\title{
Les malentendus de la technologie
}

The misunderstandings of technology

\section{Sacha Loeve et Timothée Deldicque}

\section{(2) OpenEdition \\ 1 Journals}

\section{Édition électronique}

URL : http://journals.openedition.org/artefact/2177

DOI : $10.4000 /$ artefact. 2177

ISSN : 2606-9245

Éditeur :

Association Artefact. Techniques histoire et sciences humaines, Presses universitaires du Midi

\section{Édition imprimée}

Date de publication : 6 décembre 2018

Pagination : 215-254

ISBN : 978-2-8107-0595-5

ISSN : 2273-0753

Référence électronique

Sacha Loeve et Timothée Deldicque, « Les malentendus de la technologie », Artefact [En ligne], 8|

2018, mis en ligne le 21 juin 2019, consulté le 30 novembre 2020. URL : http://

journals.openedition.org/artefact/2177 ; DOI : https://doi.org/10.4000/artefact.2177

\section{(a) $\oplus \Theta \Theta$}

Artefact, Techniques, histoire et sciences humaines est mise à disposition selon les termes de la Licence Creative Commons Attribution - Pas d'Utilisation Commerciale - Pas de Modification 4.0 International. 


\section{Les malentendus de la technologie}

\section{Sacha Loeve et Timothée Deldicque}

\section{Résumé}

Le terme actuel de technologie, en désignant directement les réalités techniques et non leur étude, les réduit au produit d'un logos appliqué qui les place dans le giron des sciences exactes et néglige leur statut de faits sociaux susceptibles d'une compréhension par les sciences humaines. Critiquant cet usage, beaucoup militent pour rétablir dans toute sa clarté la distinction entre les techniques et la technologie dans son sens originel de théorie des techniques. Cet article examine ces critiques et questionne leur volonté de dissiper les malentendus de la technologie. Il affirme que le concept de « technique » auquel on voudrait revenir est en fait postérieur à celui de technologie ; que le caractère paradigmatique de la technologie de Beckmann est discutable; et que la source de l'indétermination première comme de la surdétermination actuelle de la technologie réside dans son ambivalence irréductible entre logos de technè et technè du logos. Nous soutenons finalement qu'il existe aussi de bonnes raisons de confondre technique et technologie, comme le fait l'usage actuel, qui peut dès lors être justifié, mais à condition de le réinscrire dans un pluralisme des modes d'existence et de connaissance technologiques.

\section{Mots-clés}

analogie, applicationnisme, Johann Beckmann, histoire des techniques, philosophie des techniques, pluralisme

99 Sacha Loeve et Timothée Deldicque, « Les malentendus de la technologie », Artefact, 8, 2018, p. 215-254. 


\section{The misunderstandings of technology}

\section{Abstract}

The current word technology, by referring directly to technical realities and not to their study, reduces them to the product of applied sciences and undermines their study as social facts by the humanities. On the basis of such criticism, many call for reclaiming the distinction between technical practices, processes and artefacts on the one hand, and technology on the other, in its original sense of a theory of technè. This paper examines these criticisms and questions their willingness to dispel the misunderstandings of technology. It shows that the European concept of technique to which one would like to return is in fact posterior to that of technology; the paradigmatic role sometimes conferred on Beckmann's technology is questionable; and that the source of technology's prime indeterminacy as well as its current over-determination, lies in its irreducible ambivalence between logos of technè and technè of logos. Finally, we argue that there are also good reasons for confusing technical artefacts and technology, as is the case with current usage, which can therefore be justified, but on the condition that it be reinstated in a pluralistic conception of technological modes of existence and of knowledge.

\section{Keywords}

analogy, applicationism, Johann Beckmann, history of technology, philosophy of technology, pluralism 
$\mathrm{H}$ istoriens et anthropologues des techniques n'ont de cesse de dénoncer la confusion qui règne autour du terme " technologie ». De même que la biologie ne se confond pas avec le vivant, la sociologie avec la société ou la musicologie avec la musique, ce terme, rappellent-ils, ne désigne pas les réalités abusivement nommées " technologies " mais la discipline qui les prend pour objet. Son sens se serait donc dévoyé. Aujourd'hui, ce sont en effet les artefacts techniques et non leur connaissance que vient qualifier le suffixe «-logie». Ainsi adoubées comme "technologies", les techniques sont simultanément niées comme objets de connaissance. Leur logos ne renvoie plus au savoir que s'en ferait un sujet individuel ou collectif, mais à un mode d'existence des objets connotant pêle-mêle la science, la rationalité industrielle, la nouveauté, la modernité, l'intelligence embarquée ou le smart... Condamnant cet usage emphatique, beaucoup militent pour rétablir dans toute sa clarté la distinction entre les techniques et la théorie des techniques : la technologie.

En France, les travaux sur les significations anciennes de "technologie ", impulsés dans les années 1960, atteignent aujourd'hui un point d'orgue avec la publication, inédite en français, du texte considéré comme fondamental et structurant pour l'enseignement de la technologie, le Projet de technologie générale de Johann Beckmann ${ }^{1}$. Or sa lecture et celle des articles qui l'accompagnent invitent à relativiser la reconnaissance dont il aurait bénéficié. Même durant sa période d'institutionnalisation dans le cadre des sciences camérales de l'université allemande au début du $\mathrm{XIX}^{\mathrm{e}}$ siècle, la technologie n'a jamais accédé au rang de discipline légitime, autonome et clairement définie. Si tel est le cas, pourquoi tient-on tellement à réhabiliter son sens ancien par-delà son usage actuel ? N'aurait-on pas aussi, aujourd'hui, de " bonnes raisons " de confondre technique et technologie(s)?

L'argument principal de cet article est que la technologie n'a cessé de faire l'objet de malentendus mais que ce sont ces derniers qui en font la richesse et la fécondité. Les malentendus de la technologie ne doivent pas être levés mais plutôt élevés à la dimension du concept.

1. Johann Beckmann, Entwurf der Algemeinen Technologie, Johann Friedrich Röwer, Göttingen, 1806 ; Guillaume Carnino, Liliane Hilaire-Pérez, Jochen Hoock (dir.), La Technologie générale. Johann Beckmann, Projet de technologie générale (1806), trad. Joost Mertens et Guillaume Carnino, Rennes, PUR, 2017. 


\section{Un geste fondateur : la critique de l'applicationnisme}

Par le terme quelque peu barbare d' "applicationnisme ", nous nommons un schéma de pensée qui réduit les techniques à des applications pratiques de théories scientifiques. Ce schéma est généralement corrélé à trois thèses interdépendantes : le caractère instrumental des techniques (celles-ci n'étant que des moyens d'appliquer la science à certaines fins), leur neutralité axiologique (neutralité des moyens, moralité des fins), et le déterminisme technologique (le développement de la technique obéit à une logique autonome d'application la plus efficace possible de la science et détermine la société du dehors).

Le refus de ce schéma applicationniste a joué le rôle d'un credo fondateur dans la constitution de l'histoire des techniques comme champ distinct d'une histoire des sciences traditionnellement indifférente aux techniques. En effet, si les techniques ne sont que des applications pratiques des sciences, leur histoire se résume à leur " onde de choc "; les aspects sociaux, culturels, économiques et politiques des techniques sont secondarisés, rejetés du côté du " contexte ". L'histoire des techniques reste un produit dérivé de l'histoire des sciences.

Cependant, le positionnement initial de l'histoire des techniques diffère en France et aux États-Unis. Alors que les premières déclarations de l'École des Annales mettent l'accent sur l'interaction partielle des deux domaines de « la science " et de "la technique " ${ }^{2}$, aux États-Unis, l'émancipation de l'history of technology vis-à-vis de l'history of science est clairement revendiquée. Elle transparaît dans le titre choisi par la Society for the History of Technology (SHOT) ${ }^{3}$ pour sa revue : Technology \& Culture (TC). Ce

\footnotetext{
2. L'une des principales recommandations de Lucien Febvre et Marc Bloch était alors d'enjoindre la nouvelle discipline à réfléchir non seulement à la part de " la science » dans " la technique » mais aussi à celle de " la technique " dans " la science ", tout en replaçant « la technique » dans la diversité des activités humaines, en montrant comment elle participe de "l'histoire générale " et la modifie. Lucien Febvre, "Réflexion sur l'histoire des techniques ", Annales d'histoire économique et sociale, $\mathrm{n}^{\circ} 36,1935$, p. 531-537.

3. Sa fondation en 1958 au Massachusetts Institute of Technology (MIT) fait suite au refus de l'History of Science Society et de son influente revue Isis de s'ouvrir aux techniques.
} 
positionnement structurant pour les travaux de la $\mathrm{SHOT}^{4}$ est ensuite repris par le mouvement Science \& Technology Studies (STS) ${ }^{5}$, via notamment sa traduction dans l'histoire des techniques au sein du courant Social Construction of Technology (SCOT) $)^{6}$. Or un tel positionnement ne pouvait voir le jour que dans un univers linguistique où prédomine le vocable technology, poussant les historiens à questionner et à critiquer une référence à la connaissance (logos) qui, étant incluse dans le mot, était tenue pour évidente. À la différence du français « technique ", la confusion entre la chose et la connaissance que véhicule le mot technology a joué un rôle d'aiguillon critique, mobilisateur.

Ce rejet des connotations applicationnistes de la technology fut de plus heuristiquement fécond, dans la mesure où il débouche aussi sur une hypothèse affirmative : si en effet la technology n'est pas l'application d'une connaissance préalable, ne constitue-t-elle pas en elle-même une forme de connaissance ${ }^{7}$ ? Cette hypothèse de travail est identifiée dans les années 1980 comme un thème de recherche prometteur pour $T C^{8}$ : l'étude de la nature du savoir technique (technological knowledge) ${ }^{9}$, voire de la technique (technology) comme genre de connaissance. Malheureusement, ce thème était perçu comme trop " épistémologique » pour mobiliser pleinement les efforts des historiens ${ }^{10}$.

4. C'est ce que montre John M. Staudenmaier, Technology's storytellers, Cambridge, MIT Press, 1985, qui livre une analyse systématique de l'ensemble des publications de la revue $T C$ sur ses vingt premières années d'existence (1959-1980).

5. Bruno Latour, "La fin des moyens ", Réseaux, nº 100, 2000, p. 39-58.

6. Wiebe E. Bijker, Thomas P. Hugues, Hughes, Trevor J. Pinch, (dir.), The social construction of technological systems, Cambridge, MIT Press, 1987.

7. Cette proposition va plus loin que n'iront les courants STS et SCOT des années 1980-90, qui ne s'intéressent guère à explorer cette voie.

8. Articulée en 1985 par J. Staudenmaier, op. cit., p. 120, elle avait déjà été formulée dans les lignes de TC par Edwin T. Layton, "Technology as knowledge", Technology \& Culture, vol. 15, n 1 , 1974, p. 31-41. Voir aussi la reprise des idées de Layton par Ann JoHnson, « Revisiting technology as knowledge ", Perspective on Science, vol. 13, n 4, 2006, p. 554-573.

9. Wybo Houkes, "The nature of technological knowledge”, in Anthonie Meijers (dir.), Philosophy of technology and engineering sciences, New York, Elsevier, 2009, p. 309-350.

10. L'ouvrage de Walter G. VInCENTI, What engineers know and how they know it, Baltimore, Johns Hopkins University Press, 1990, semble faire exception, mais Vincenti justement, n'est pas historien de formation, mais d'abord ingénieur, et travaille sur l'histoire de son domaine, l'aviation. De plus, son approche du savoir technique relève moins d'une épistémologie des techniques au sens large que d'une épistémologie de la conception dans le contexte des sciences de l'ingénieur. Le thème du savoir technique a aussi été pris en charge dans le cadre de recherches sur les politiques d'innovation par Wendy FaulKner, "Conceptualizing knowledge used in innovation", Science, 
Cependant, en France, cette question animait déjà, indépendamment et par d'autres voies - plus épistémologiques que celles de l'École des Annales - le séminaire sur "Les commencements de la technologie » organisé de 1963 à 1965 sous la tutelle de George Canguilhem à l'Institut d'histoire des sciences et des techniques (IHST). Or Canguilhem critique le schéma applicationniste. Prenant le contre-pied de Bachelard, il explique qu'une machine n'est pas un " théorème réifié "; que même une technique " scientifique » n'est jamais déductible d'une théorie pure, mais toujours rendue possible par un emprunt que les sciences font aux techniques qui leur préexistent ${ }^{11}$.

\section{L'exhumation du concept ancien de technologie}

Le séminaire "Les commencements de la technologie " débouche sur la publication en 1966 d'un article tenu pour fondateur des travaux d'histoire de la technologie ${ }^{12}$. Si la voie d'entrée n'est pas la technology comme genre de connaissance mais la technologie comme science des techniques, les problèmes se rejoignent. Se donnant pour tâche de retracer l'histoire

Technology and Human Values, vol. 19, n ${ }^{\circ}$ 4, 1994, p. 425-458. Celle-ci propose une catégorisation des connaissances portant 1) sur le monde naturel, 2) sur la pratique de conception inventive, 3) sur les procédures de $\mathrm{R} \& \mathrm{D}$ expérimentale, 4) sur les produits finaux, et 5) sur la connaissance elle-même, mais comme le titre l'indique, la focale est placée sur les connaissances entrantes plutôt que sur les connaissances issues des techniques. Pour le reste, le thème a surtout mobilisé des philosophes. Par exemple Günter Ropohl, "Knowledge types in technology", International Journal of Technology and Design Education, vol. 7, n ${ }^{\circ}$ 1, 1997, p. 65-72, dont la typologie comporte : règles structurales (qui régissent l'assemblage des composants d'un système), lois technologiques (qui opérationnalisent des lois naturelles), règles fonctionnelles (qui régissent les relations des moyens et des fins dans des circonstances données), savoir-faire techniques (implicites et tacites), et compréhension sociotechnique (qui porte sur les interactions entre artéfacts, environnement et société). Mais sa perspective reste très instrumentale et assimile la technologie, par différence d'avec la simple technique, à l'ingénierie. Ou encore Marc J. DE VRIEs, "The nature of technological knowledge: Extending empirically informed studies into what Engineers know”, Techné: Research in Philosophy and Technology, vol. 6, n ${ }^{\circ}$, 2003, p. 117-130, insiste sur la distinction entre connaissance scientifique, descriptive et visant la vérité, et connaissance technologique, normative et visant l'efficacité, mais cette dichotomie est critiquée pour son simplisme par W. HoukEs, art. cit.

11. Georges Canguilhem, "Machine et organisme " (1947), in Connaissance de la vie, Paris, Vrin, 1965 [1952], p. 101-127. Voir aussi Jan Sebestik « Postface/préface. Les commencements de la technologie ", Documents pour l'histoire des techniques, 2007, p. 123-133.

12. Jan Sebestik, Jacques Guillerme, "Les commencements de la technologie ", Thalès, t. XII (1966), 1968, p. 1-72. Republié dans Documents pour l'histoire des techniques, 2007, p. 49-122. 
de la légitimation du savoir technique comme science, les auteurs Jacques Guillerme et Jan Sebestik exhument la signification oubliée de " technologie " comme "discours sur les techniques " - logos de la technè - au sens d'étude, de théorie voire de science des techniques. Situant les commencements de la technologie comme projet disciplinaire au milieu du $\mathrm{XVIII}^{\mathrm{e}}$ siècle, ils en perçoivent les prémisses dans l'Encyclopédie de Diderot et D'Alembert (1751-1772), qui a l'ambition de constituer une langue universelle des arts et métiers et en propose une description classifiée alphabétiquement par matières et métiers s'appuyant sur de nombreuses planches de dessins ${ }^{13}$. Cependant les encyclopédistes n'emploient pas le terme technologie ${ }^{14}$.

C'est outre-rhin, affirment Guillerme et Sebestik, que la technologie acquiert une véritable existence disciplinaire. En 1728, le philosophe Christian Wolff définit la "Technica ou Technologia"15 " comme "science des arts et des œuvres de l'art (scientia artium \& operum artis), ou si l'on préfère, science des choses que les hommes produisent par le travail des organes du corps, principalement par les mains ${ }^{16}{ }$. Cependant pour Wolff " la Technologia emprunte ses principes à la Physique, surtout expérimentale ", aussi " celui qui veut rendre raison des choses produites par l'art doit recourir à la Physique ${ }^{17}$ ". C'est pourquoi il faut disposer et traiter la technologie après la physique. Par conséquent, loin d'envisager la fondation d'une science autonome qui produise des connaissances sui generis, Wolff dessine un partage entre le comment physique et le pourquoi technologique : la connaissance de la structure et du fonctionnement des objets fabriqués est du ressort de la physique, celle des raisons et des fins des objets fabriqués relève de la technologie. La technologie ne se distingue de la physique que par son objectif de rendre compte des règles de l'art, en utilisant les principes déjà établis par cette dernière. Pour rendre compte de la multiplicité des arts, Wolff en appelle certes à une " histoire précise des arts ", mais celle-ci peut et même doit attendre, car « il y a d'autant moins

13. L'Encyclopédie compte 28 volumes, 17 de discours et 11 volumes de planches.

14. À l'exception de l'article "Catalogue » qui concerne la classification des ouvrages dans les bibliothèques. J. Sebestik, J. Guillerme, art. cit., p. 2.

15. Wolff introduit le sujet en utilisant les deux termes, même s'il parle ensuite de technologia pour définir cette science.

16. Christian Wolff, Philosophia rationalis sive Logica, \$71. Cité par J. Sebestik, J. Guillerme, art. cit., p. 29.

17. C. WolfF, op. cit., $\$ 113$. 
à discourir de ces [choses] qu'elles supposent la science déjà connue et vue avec netteté18 ». Pour Guillerme et Sebestik, Wolff propose une " théorie de l'application » devant être portée, selon lui, par un "troisième homme » qui réunirait en lui la science et l'art ${ }^{19}$. Mais on peut aussi interpréter son point de vue comme une tentative de réduction instrumentale des techniques aux sciences qui annonce les beaux jours de l'applicationnisme moderne.

C'est dans ce contexte que Johann Beckmann commence à enseigner la technologie à l'université de Göttingen au début des années 1770 et publie l'Anleitung zur Technolgie en 1777. Bien qu'il s'inscrive dans le prolongement de l'Encyclopédie, qu'il cite abondamment dans son introduction, il souhaite fonder une véritable discipline scientifique qui s'en distingue par le traitement systématique de son objet en fonction " de l'égalité ou la similitude dans le procédé et dans les raisons [Gründe] sur lequel il repose ${ }^{20} »$. L'ambition de Beckmann est partiellement réalisée lorsqu' en 1806 il publie l'Entwurf der algemeinen Technologie, par lequel il entend « dresser un catalogue de tous les buts poursuivis par les artisans et les artistes au cours de leurs diverses opérations » appelé " technologie générale ${ }^{21}$ ".

222 Cependant, alors que la discipline technologie se développe en Allemagne, l'article de Guillerme et Sebestik et les travaux d'histoire de la technologie suivants montrent que le projet disciplinaire de la technologie s'épuise au milieu du XIX ${ }^{\mathrm{e}}$ siècle $^{22}$. Guillaume Carnino observe que cette " évanescence " est concomitante de l'avènement de "la science " comme catégorie hégémonique ${ }^{23}$. Dès lors, la promotion du schéma applicationniste est corrélative d'un basculement du logos de technologie. La technologie ne fait plus "référence au logos antique mais à la "-logie" propre aux sciences modernes " et s'apparente à une "rationalisation scientifique de

18. Ibid, $\$ 114$.

19. J. Sebestik, J. Guillerme, art. cit., p. 23.

20. Johann Beckmann, Anleitung zur Technologie. Cité par J. Sebestik, J. Guillerme, art. cit., p. 41.

21. Johann Beckmann, Projet de technologie générale, op. cit., p. 68.

22. Par exemple Joost Mertens, "Le déclin de la technologie générale : Léon Lalanne et l'ascendance de la science des machines ", Documents pour l'histoire des technique, n 20, 2011, p. 107-117. 23. Guillaume CARNINO, «Les transformations de la technologie : du discours sur les techniques à la "techno-science" ", Romantisme, n' 150, 2010, p. 75-84. Guillaume Carnino, L'invention de la science. La nouvelle religion de l'âge industriel, Paris, Seuil, 2015. 
la technique ${ }^{24} »$. Elle ne désigne plus le discours sur les techniques mais rassemble "procédures industrielles et produits matériels ». Le projet disciplinaire de la technologie s'écroule sous son propre poids et se fragmente en une multitude de nouvelles disciplines dites " appliquées ${ }^{25}$ ", dont les fruits sont finalement identifiés aux " technologies" au sens contemporain - et confus - du terme.

\section{La critique de l'anglicisme : un faux problème qui en cache un vrai}

Une critique souvent formulée contre l'usage contemporain de " technologie " est qu'il s'agit tout simplement d'un anglicisme. Le technology nord-américain aurait remplacé et déclassé les termes anglais de technique et de technics ${ }^{26}$ avant de s'imposer au monde entier via l'hégémonie

24. G. Carnino, L. Hilaire-Pérez, "Qu'est-ce que la technologie ? Jalons pour une histoire longue d'un concept oublié ", in G. Carnino, L. Hilaire-Pérez, J. Нoock, op. cit., p. 13-36, p. 29-30.

25. «La technologie, c'est alors les aventures dans l'industrie, de la science appliquée », J. SEBESTIK, J. Guillerme, art. cit., p. 3. «Les techniques retournent à l'arrière-plan qu'elles avaient un moment quitté, l'idéologie de la science appliquée va s'installer ». François Sigaut, « Préface, Haudricourt et la technologie ", in Georges HaUdricourT, La technologie science humaine. Recherche d'histoire et d'ethnologie des techniques, Paris, MSH, 1987, p. 24. Marx peut alors écrire que " [le] principe [de la grande industrie] qui est de dissocier tout procès de production, pris pour lui-même, et sans aucun égard pour la main humaine en ses éléments constitutifs, a créé cette science toute moderne qu'est la technologie. Les figures bigarrées, éparses et sclérosées du procès social de production se sont décomposées en applications méthodiques et conscientes des sciences naturelles, systématiquement séparées les unes des autres selon le rendement recherché ». Karl Marx, Le capital. Critique de l'économie politique. Livre premier. Le procès de production du capital (1867), $4^{\mathrm{e}}$ édition allemande (1890), Paris, PUF, 1993 [1983], p. 546. Notons que si la technologie désigne encore la science de la production industrielle chez Marx - lecteur d'Andrew Ure comme de Johann Heinrich M. von Poppe (auteur d'une Geschichte der Technologie publiée à Göttingen en 1807 et professeur de Technologie à Tübingen à partir de 1818, il est cité dans le troisième livre du Capital) - elle désigne déjà en 1867 la production industrielle elle-même comme cela est visible dans plusieurs passages du Capital, par exemple : "De toutes les marchandises, ce sont les marchandises de luxe proprement dites qui sont les moins significatives pour comparer la technologie de différentes époques de production " (p. 206, note 5). Ou encore : " une histoire critique de la technologie [...] prouverait combien il est rare qu'une invention quelconque, au XviII ${ }^{\mathrm{e}}$ siècle, soit le fait d'un seul individu " (p. 417, note 89 ).

26. Technics est employé un temps, notamment par Lewis Mumford, Technics and Civilization, New York, Harcourt, 1939, puis tombe en désuétude, y compris chez Mumford, qui utilise technology après-guerre. 
économique et culturelle des États-Unis après-guerre ${ }^{27}$. Guillerme parle d'une "contamination "franglaise" " après la Seconde Guerre mondiale, condamnée par le Comité d'études des termes techniques de l'Académie française. Pour François Sigaut, la technologie est devenue « une espèce de superlatif savant, ou pédant, de technique ». Les deux auteurs s'appuient sur la chronique Langage du Monde tenue par le romancier Jacques Cellard en 1980 pour qui cet "abus prétentieux " fait " plus noble, plus chargé de science » ajoutant que « quatre-vingt-quinze fois sur cent l'emploi de technologie et technologique n'est qu'un faux sens emphatique et lourd $»^{28}$.

Se contenter de dénoncer l'hégémonie d'un anglicisme snob paraît trop simple, assez oiseux (tel n'est pas d'ailleurs ce que font Guillerme ou Sigaut). Au-delà de la question du mot et de la domination linguistique qu'il traduit se pose le problème du concept qui en surdétermine le sens. À ce titre, l'historien de la culture américaine Leo Marx qualifie le concept actuel de technology de "dangereux " en raison de la réification du social dont il est, selon lui, porteur ${ }^{29}$. Si en effet la technique est ce qui médiatise les relations des humains entre eux et avec la nature, la technologie est ce qui rend compte de la logique et du sens de ces relations, ce qui correspond à son acception ancienne. Mais dès lors que technology vient désigner un domaine d'objets et de relations entre objets, le concept occulte les dimensions humaines de l'activité et de la pensée techniques en les présentant sous les aspects objectifs d'un ordre des choses - il les réifie.

Analysant son émergence récente comme mot-clé de la culture nord-américaine, L. Marx soutient que technology serait venu combler un vide sémantique creusé par la collusion de deux facteurs. Idéologique, d'une part : il vient nommer un progrès qui ne peut plus être légitimé politiquement en instaurant un nouveau sacré. Substantiel, d'autre part : il reflète la systématisation et le déploiement des machines à des échelles d'espace et de temps dépassant l'ordre de grandeur de ce qui est humainement représentable

27. Remarquons qu'il faudrait aussi étudier par souci de symétrie ce qu'il en est allé des mots de technique et de technologie dans la langue russe pendant la période soviétique.

28. Jacques Guillerme, article "Technologie » de l'Encyclopedia Universalis (édition 1995), republié dans E-Phaïstos, III-1, 2014, p. 84-92, p. 84 ; François Sigaut, "Préface, Haudricourt et la technologie ", in André-Georges Haudricourt, La technologie science humaine. Recherches d'histoire et d'ethnologie des techniques, Paris, MSH, 1987 p. 9-32, p. 9 ; Jacques Cellard, "Chronique Langage ", Le Monde, 23/04/1980.

29. Léo Marx, "Technology. The emergence of a hazardous concept", Technology and Culture, vol. 51, n 3, 2010, p. 561-577. 
(des microprocesseurs aux réseaux planétaires et satellitaires). Alors qu'une expression comme " les arts industriels " conservait un lien avec l'activité technique concrète et vécue des métiers, le mot technology rompt ce lien pour instaurer une nouvelle construction de la réalité dans laquelle la technique est dématérialisée et le social réifié, s’imposant comme un donné objectif qui aurait toujours existé. Ainsi le concept actuel et courant de technologie présente, écrit-il, cette " circularité ou réflexivité impliquée dans la construction sociale de mots-clés qui - comme culture, industrie, démocratie - viennent servir de marqueurs historiques pour des périodes où ils acquièrent des significations nouvelles et fondamentalement altérées ${ }^{30}$ ".

Dès lors, on pourrait penser que la réhabilitation du sens européen de technologie comme théorie des techniques pourrait nous préserver des « dangers » d'un sens nord-américain dévoyé qui la confond avec son objet (et l'y engloutit). Il suffirait de réhabiliter la technologie comme théorie des techniques pour se réapproprier du même coup " la technique " comme objet de connaissance et de culture constitutif de notre humanité.

Hélas, l'affaire se complique quand on prend en compte un aspect méconnu mais décisif de l'histoire du technology nord-américain, établi par Éric Schatzberg ${ }^{31}$. Ce dernier rappelle d'abord que technology existe dans une acception similaire au sens allemand et français dans la langue anglaise savante du XIX ${ }^{\mathrm{e}}$ siècle : " the study of practical arts». Mais il soutient que ce n'est pas de ce dernier que dérive le technology anglais moderne au sens de " the artifacts, material means of production and processes of industrial civilization". Ce glissement sémantique ne s'explique pas par une migration du concept oublieuse de ses origines européennes, mais par la traduction et la reprise dans le contexte américain d'un nouveau concept : die Technik, c'est-à-dire la technique.

\section{Le piège de " la technique"}

Le mot " technique " apparaît dans les langues européennes modernes à la fin du XviII ${ }^{\mathrm{e}}$ siècle, soit comme adjectif (les " termes techniques ${ }^{32}$ ),

30. Ibid., p. 575 (notre traduction; italiques de l'auteur).

31. Éric Schatzberg, "Technik comes to America: Changing Meanings of Technology before 1930", Technique and Culture, vol. 47, n 3, 2006, p. 486-512.

32. Dont l'étude se nomme d'ailleurs " technologie ", au sens de la technologia (technè du logos). 
soit comme nom transitif (" la technique de X » au sens de la "méthode de $X »)$, mais il n'apparaît comme substantif intransitif ("la ou les technique(s)") que durant la seconde moitié du $\mathrm{XIX}^{\mathrm{e}}$ siècle, époque où le mot Technologie est tombé en désuétude. " $L a$ technique " apparaît d'abord en Allemand ("die Technik»), puis en Français à la fin du XIX siècle, où son emploi se généralise durant l'entre-deux-guerres, pas avant ${ }^{33}$.

C'est Alfred Espinas qui a sinon introduit ${ }^{34}$, du moins milité pour l'introduction du substantif intransitif « la ou les technique(s) » dans la langue française. En 1897, il écrit : " nous pourrions donner aux arts utiles le nom de techniques pour les distinguer des arts qui tendent à produire l'émotion esthétique. Ce mot de technique a malheureusement chez nous un sens assez restreint ; nous disons la technique de l'enseignement, la technique de telle ou telle fabrication et nous désignons ainsi les procédés opératoires ou, en général, les parties spéciales des arts industriels (ou d'autres qu'on leur assimile) plutôt que ces arts eux-mêmes ${ }^{35}$ ». Espinas a probablement en tête le terme allemand, puisqu'il se réfère souvent à Ernst Kapp, auteur en 1877 du Grundlinien einer Philosophie der Technik, le premier livre titré " philosophie de la technique ".

Die Technik désigne alors les arts industriels comme un tout cohérent. Schatzberg raconte qu'il est promu comme maitre mot de la culture ingénieur par la Verein Deutscher Ingenieure (Association des ingénieurs allemands, VDI, fondée en 1856). Die Technik est l'objet de l'ingénierie, non de la technologie. La VDI est alors préoccupée par la relation entre Technik et Kultur. La question suscite moult débats dans un contexte d'ascension sociale et de promotion culturelle des ingénieurs. Se noue dans ce contexte une alliance entre ingénieurs et philosophes défendant la dignité culturelle de la Technik. Une "philosophie de la technique» voit alors le jour, avec des auteurs comme Ernst Kapp, Eberhard Zschimmer, et Friedrich Dessauer. Leurs philosophies instaurent "la technique " comme phénomène global

33. Par exemple, en 1907, Bergson dans L'évolution créatrice utilise en abondance des termes comme " artificiel ", " construction ", " industrie ", " instruments ", " inventions ", " fabrication ", " fonctionnel », « machine », " manipulation », " manufacture », « mécanismes » ou " outils ", mais on n’y trouve aucune occurrence de "la technique ", contre deux occurrences seulement en 1932 dans Les deux sources de la morale et de la religion - deux ouvrages traitant pourtant centralement de ce qu'il est aujourd'hui convenu d'appeler "philosophie de la technique ".

34. Selon le dictionnaire d'André Lalande, Vocabulaire technique et critique de la philosophie, Paris, Alcan, 1902-1932.

35. Alfred Espinas, Les origines de la technologie, Paris, Alcan, 1897, p. 7 (italiques de l'auteur). 
reliant l'humain comme être de culture aux deux pôles de la vie organique et des idéalités. Toutes insistent sur la dimension spirituelle de la technique d'un côté, et sur sa dimension organique, inconsciente, de l'autre ${ }^{36}$. Ainsi, "la technique " est-elle, en ce sens, une invention de philosophes... en milieu ingénieur.

Selon Schatzberg, c'est l'économiste et sociologue Thorstein Veblen (18571929) qui fait la charnière entre l'allemand die Technik et l'anglais technology, qu'il aurait introduit et popularisé aux États-Unis. Veblen a lu et commenté les auteurs de l'économie historique allemande, en particulier Gustav Schmoller et Werner Sombart, qui intègrent die Technik à leur lecture du capitalisme moderne. Et c'est après ces lectures allemandes, vers 1900, que technology devient un mot-clé de l'arsenal conceptuel de Veblen (à partir de The Theory of Buisiness Enterprise, 1904).

Ce penseur décapant, prônant l'institution d'un "Soviet des ingénieurs " aux États-Unis contre le "sabotage » de l'économie par le capitalisme financier de la business enterprise, devient très célèbre, notamment comme éditorialiste ; ses idées inspirent le "Parti technocratique " après la crise de 1927. Selon Schatzberg, Veblen aurait eu besoin d'un concept assez englobant pour dénoter à la fois l'état de l'art du développement matériel

36. Ainsi lit-on dès les premières pages de l'ouvrage d'Ernst Kapp, Grundlinien einer Philosophie der Technik, Brunswick, Westermann, 1877, que le problème de la philosophie de la technique est celui de la culture : comment l'homme se (re)connaît et se symbolise en transformant le monde. La réponse de Kapp est la "projection d'organe ", qui comporte deux étapes : 1) l'homme projette inconsciemment son fonctionnement moteur, énergétique et nerveux dans des outils, machines et réseaux ; 2) l'homme les utilise comme des modèles de connaissance du monde tout en s'y reconnaissant comme leur origine inconsciente. Kapp voit dans ce double mouvement de projectionreconnaissance l'origine de la conscience au niveau individuel comme de la culture au niveau collectif : la culture est la conscience du corps de l'espèce humaine. Sa philosophie est anthropocentriste, organiciste et matérialiste : l'esprit est le corps se connaissant lui-même par extériorisation de son action, de même que la culture est la civilisation matérielle s'organisant de plus en plus consciemment (voir la traduction française par Grégoire Chamayou, Ernst Kapp, Principes d'une philosophie de la technique, Paris, Vrin, 2007). Eberhard Zschimmer (Philosophie der Technik: vom Sinn der Technik und Kritik des Unsinns über die Technik, Jena : Mittler 1914 ; Technik und Idealismus, Jena, Jenaer Volksbuch, 1920) défend une conception de la technique comme « outil de la culture » et du progrès technique comme progrès culturel à condition qu'il soit éclairé et pris en main par l'État contre l'anarchie du laisser-faire économique. Quant à Friedrich Dessauer (Philosophie der Technik. Das Problem der Realisierung, Bonn, Cohen, 1927), il développe une approche néoplatonicienne de la technique visant à dépasser le kantisme : la technique fait passer les choses en soi dans le monde phénoménal et les rend connaissables et sensibles. Les possibles techniques préexistent en soi ; l'activité d'invention les fait passer à l'existence, prolongeant ainsi la création divine. 
d'une société donnée et son équipement immatériel, c'est-à-dire le savoir collectif dont une société dispose concernant les ressources, leurs procédés de transformation et de valorisation au service du bien commun. Ce faisant, il construit un concept sophistiqué, politiquement engagé et nondéterministe de technology, opérant un syncrétisme entre le Technik allemand et l'ancien technology dans le cadre d'un projet socialiste.

Mais le concept est ensuite repris dans un sens acritique, laissant de côté le contenu politique qu'il avait chez Veblen. Schatzberg rejoint alors les analyses de L. Marx. Il documente notamment l'analyse de l'usage « apologétique plutôt que critique " de technology chez l'historien progressiste Charles Beard. Au sortir de la guerre, le concept s'est finalement transmué en un étrange hybride : à la fois fruit nécessaire de la science pure ${ }^{37}$ et force autonome faisant croître l'économie selon une nécessité organique ${ }^{38}$. D'après Schatzberg c'est cette reprise oublieuse d'un concept politiquement chargé qui a donné le technology actuel, dont l'usage, affirme-t-il, est aussi banal qu'incohérent, puisqu'il constitue un mixte mal composé de technological knowledge et de die Technik, phénomène global à la fois organique et calculatoire, monstre étrange fait de vie et d'intelligence.

Si le mot technology dérive de l'allemand die Technik, il n'a pas simplement remplacé l'anglais technique $e^{39}$. Ce n'est donc pas en rétablissant le concept

37. On songe au célèbre rapport de Vannevar Bush, Science, the endless frontier, Office of scientific research and development, 1945. Sur ce point, il faudrait compléter l'analyse de Schatzberg en tenant compte du double mouvement de promotion de techniques basées sur la science (sciencebased) et de sciences hautement technicisées (la "Big science ») engagé dans le cadre de la réorganisation des sciences sous la Guerre froide, qui installe un régime qualifié aujourd'hui de " technoscientifique ". Voir Bernadette Bensaude Vincent, Les vertiges de la technoscience, Paris, La Découverte, 2009, part. I, chap. 2.

38. On songe au modèle économique de Robert Solow, qui milite dans les années 1950 pour que les économistes reconnaissent le progrès technique comme moteur de la croissance économique. Moteur invisible, puisqu'il ne peut pas être lui-même expliqué par l'économie, mais simplement mesuré par le différentiel entre investissements et profit. Pour la science économique d'après-guerre, le progrès technique devient la boîte noire qui explique la croissance sans qu'on ait même besoin de comprendre comment il y contribue. Voir Verena Halsmeyer, "From long-run utopia to technical expertise: Solow's growth model as a multipurpose design", The Center for the History of Political Economy (CHOPE) Working Paper, n 2014-09, 2014, 25 p.

39. Qui n'a d'ailleurs jamais désigné " la ou les technique(s) » comme substantif intransitif. L'expression employée était useful arts. Dans les langues européennes, les " arts ", proviennent du latin ars (mixte d'opératio et de thêoria) qui traduit le grec ancien technè, et que l'on retrouve dans " technologie ». En revanche le terme anglais de technique, qui dénote une méthode, une recette, un talent, une habileté, une astuce, une ruse voire un «truc ", tient moins du grec ancien technè que du 
de « technique » dans son prétendu sens originel que l'on pourra ouvrir de nouveaux horizons pour la technologie. Le concept de technique auquel on voudrait revenir en rétablissant la distinction technique/technologie est en réalité postérieur à la technologie, et complice de son sens actuel. Là encore, le faux problème (l'idée que le sens actuel de technologie aurait destitué le sens traditionnel de technique) en cache un vrai : le piège du substantif «la technique».

Ce piège apparaît béant lorsqu'on s'attache au chapitre sombre de l'histoire du concept de Technik allemand durant l'entre-deux-guerres, où la double dimension vitale et spirituelle du nouveau concept est mise au service du national-socialisme dans le sillon d'Ernst Jünger puis d'Ostwald Spengler ${ }^{40}$ et de ses disciples nazis Manfred Schröter ${ }^{41}$ et Eugen Diesel ${ }^{42}$. La Technik apparaît chez ces auteurs comme le summum de cette " étrange combinaison " dont parle Schatzberg pour qualifier la technology contemporaine : un hybride de nécessité organique et de science appliquée. Chez Spengler, son caractère organique est interprété comme force d'expansion de l'espace vital, « tactique vitale » dans la lutte pour la survie des peuples; son caractère spirituel est interprété comme rationalité calculatoire, calcul optimal des moyens, destin d'une science toujours-déjà appliquée, « outil

latin tardif techna qui signifie artifice, fourberie, moyen de tromperie, sophisme, procédé langagier, figure de rhétorique, avec une connotation péjorative. Techna s'apparente aussi à la mètis grecque, ruse ou détour, vertu d'Ulysse ou de Dédale, aux connotations plus ambivalentes (parfois positives). Sur techna, voir Jacques Guillerme, "Les liens du sens dans l'histoire de la technologie ", Cahiers STS, $\mathrm{n}^{\circ}$ 2, 1984, p. 23-29, republié dans Jacques Guillerme, L'art du projet, Wavre, Mardaga, 2008, p. 17-22, p. 18.

40. Ernst JüNGer, Die totale Mobilmachung (La mobilisation totale), in E. JüNGer, Krieg und Krieger, Berlin, Junker und Dünnhaupt, 1930 ; Der Arbeiter. Herrschaft und Gestalt (Le travailleur. Domination et Forme), Hamburg, Hanseatische Verlagsanstalt, 1932. Ostwald Spengler, Der Untergang des Abendlandes (le déclin de l'occident) - Umrisse einer Morphologie der Weltgeschichte, Vienne, Munich, Beck Verlag, 1918-1922 ; Der Mensch und die Technik. Beitrag zu einer Philosophie des Lebens, Munich, Beck Verlag, 1931. Spengler critique le régime nazi mais porte au pinacle le fascisme italien et les nazis reprennent ses slogans. Le cas de Jünger est plus complexe. Nationaliste militant et ancien combattant de la Première Guerre mondiale, il refuse les appels de pieds des nazis et les critique même ouvertement. Mais il n'est pas inquiété par eux en raison de la sympathie qui lui voue Hitler. Il se retire ensuite dans la forêt bavaroise en ermite psychédélique, et meurt centenaire.

41. Manfred Schröter, "Philosophie der Technik", in A. Baeumler und M. Schröter (eds.), Handbuch der Philosophie (chap. 43), Oldenbourg, München und Berlin, Druck und Verlag von R. Oldenbourg, 1934 ; Deutscher Geist in der Technik, Köln, Schaffstein, 1935.

42. Fils de l'inventeur du moteur Diesel, Eugen Diesel, Das Phänomen der Technik, Zeugnisse, Deutung und Wirklichkeit, Berlin, VDI-Verlag, 1939. 
de la technique ", comme le dira Heidegger. Destin vital et destin spirituel, force vitale inconsciente et calcul surrationnel coïncident en un phénomène monolithique, menaçant - "faustien ", dit Spengler.

Un autre trait caractéristique du concept de Technik comme phénomène global est qu'il est décrit soit de manière fonctionnaliste (des moyens optimaux pour des fins : la Zweckrationnalität théorisée par Max Weber), soit comme un court-circuit de ce fonctionnalisme : les moyens deviennent leurs propres fins, la technique devient autonome, autotélique ; elle ne vise que son propre accroissement. Et les auteurs de ces années sombres ajoutent souvent que c'est le propre de la technique "moderne " (caractérisée par le Gestell heideggérien). Ce phénomène global, menaçant, vital, et autotélique de "la technique » aura ses thuriféraires : ceux qui acceptent le "pacte faustien " que Spengler appelle de ses voux, ses " ambigus " (Jünger, Heidegger), et ses critiques radicaux, tels Günter Anders, Hans Jonas, Michel Henri ou Jacques Ellul en France. Mais les thuriféraires comme les critiques acceptent une vision à peu près similaire de " $l a$ technique ": celle d'un phénomène monolithique et par essence ambivalent, car doublement inscrit dans les processus vitaux et dans une rationalité calculatoire désincarnée.

Bref, si la technologie entendue comme théorie des techniques peut trouver une quelconque pertinence aujourd'hui, ce ne peut être qu'en résistant à l'unification de son objet, en affirmant et en cultivant bien plutôt la pluralisation des logoï de technè.

\section{La remotivation du sens ancien dans les sciences humaines ${ }^{43}$}

Les travaux d'histoire de la technologie écrits à la suite de l'article de Guillerme et Sebestik ont lancé dans une certaine mesure une telle

43. Nous empruntons la notion de "remotivation " à la linguistique saussurienne. En vertu du caractère arbitraire du signe linguistique, le signifiant (l'image acoustique) n'a aucun rapport naturel avec son signifié (le concept) : le signe est dit immotivé. Cependant, le signe peut être dit relativement motivé lorsqu'il fait transparaître dans le signifiant une analogie perceptible avec le signifié. En linguistique dite synchronique, la motivation relative s'opère selon des procédés morphologiques de composition (" dix-neuf " est plus motivé que "vingt ") ou de dérivation par ajout de préfixe ou de suffixe (" poirier " est plus motivé que "poire ») reconnus par une communauté linguistique. Dans les domaines scientifiques et techniques, les néologismes et les termes composés s'inspirant du grec 
pluralisation. Capitalisant sur la redécouverte du sens ancien de technologie, ils l'étendent à d'autres époques ${ }^{44}$ et à d'autres pratiques ${ }^{45}$, quand bien même l'usage du mot n'y apparait pas, tout en convergeant pour identifier dans le projet de Beckmann la manifestation du principe nodal du savoir technique et se faisant de la technologie : l'analogie d'opération ${ }^{46}$. Ils remotivent et se réapproprient le sens ancien de technologie contre son sens moderne et étayent ainsi la critique du schéma applicationniste, auquel ils opposent une définition de la technologie comme " théorie générale des opérations transversales " aux domaines techniques, suivant le schème de l'analogie ${ }^{47}$.

Cette remotivation du sens ancien de technologie se lit également chez les anthropologues et ethnologues du $\mathrm{xx}^{\mathrm{e}}$ siècle s'inscrivant dans la lignée

ancien sont en général relativement motivés, à l’instar du terme " technologie ". En linguistique dite diachronique, le phénomène de motivation relative s'appréhende à travers le temps. Ainsi un signe peut être le lieu d'une démotivation, ou au contraire d'une remotivation lorsque ses locuteurs estiment que son lien avec sa motivation première s'est perdu, comme c'est le cas pour le terme " technologie ». Voir par exemple Marie-José Reichler-BÉGUelin, " Motivation et remotivation des signes linguistiques ", Revue de Philologie, de Littérature et d'Histoire Anciennes, 1991, vol. 65, $\mathrm{n}^{\circ} 1$, p. 9-18.

44. C'est notamment le cas de l'ouvrage collectif dirigé par Hélène Vérin et Pascal DubourgGlatigny (dir.), Réduire en art. La technologie de la Renaissance aux Lumières, Paris, MSH, 2008, qui porte sur le genre littéraire de la réduction en arts, consistant à rassembler des savoirs pratiques puis à les réduire en principes en les ordonnant à l'aide des mathématiques et de la rhétorique.

45. Liliane Hilaire-Pérez, "L'histoire intellectuelle des techniques au Centre d'histoire des techniques et de l'environnement du Conservatoire national des arts et métiers ", Revue de synthèse, vol. 130, $\mathrm{n}^{\circ} 1,2009$, p. 147-164, décrit la tradition technologique comme " genre littéraire " caractérisé et affirme son inscription dans la longue durée : "L'origine de ce genre littéraire remonte à la tradition cicéronienne de formalisation des pratiques, notamment juridiques, poursuivie au Moyen Âge, élargie à l'art de la guerre, à l'agriculture et à la mécanique, bien que toujours pour un public restreint " (p. 149). Les travaux de Liliane Hilaire-Pérez sur la ville de Londres au XviII ${ }^{\mathrm{e}}$ siècle, montrent qu'une « technologie » prend forme au sein des pratiques artisanales au contact du monde économique et industriel en transformation, dans un contexte d'émergence d'un espace public de la technique. Liliane Hilaire-Pérez, La pièce et le geste. Artisans, marchands et savoir technique au XVIII siècle, Paris, Albin Michel, 2013.

46. L. Hilaire-Pérez et G. Carnino parlent de l'analogie comme de la " figure clé de la technologie ». G. Carnino, L. Hilaire-Pérez, art. cit., p. 18. Nous y reviendrons.

47. G. Carnino, L. Hilaire-Pérez, art. cit., p. 21-22. Les auteurs s'appuient sur les travaux d'Hélène Vérin. Voir Hélène VérIn, "La technologie : science autonome ou science intermédiaire ? ", Documents pour l'histoire des techniques, n 14, 2007, p. 134-143. Cet intérêt pour l'analogie dans les techniques s'est traduit par la publication d'un ouvrage collectif : Sophie De Beaune, Liliane Hilaire-Pérez, Koen Vermeir (dir.), L’analogie dans les techniques, Paris, CNRS Éditions, 2017. 
des travaux de Marcel Mauss ${ }^{48}$, André-Georges Haudricourt ${ }^{49}$ et François Sigaut $^{50}$ - chacun respectivement élève du précédent, tenants de la " technologie science humaine " (ou " technologie culturelle»). Pour eux, la technologie porte d'abord sur l'homme, qui ne saurait être étudié abstraction faite de ses techniques. Selon Sigaut, il faut « étudier les techniques pour elles-mêmes " - in fine, pour comprendre l'homme ${ }^{51}$. Selon Haudricourt, " c'est le point de vue humain, de la fabrication et de l'utilisation par l'homme qui est essentiel » et même "plus essentiel que les autres ${ }^{52}$ " dans l'étude des techniques.

Au motif de ne pas laisser la technologie aux scientifiques et ingénieurs mus par le schéma applicationniste, Haudricourt et Sigaut la revendiquent comme pré carré des sciences humaines. Or la focalisation sur l'homme comme producteur et utilisateur ${ }^{53}$ fait courir le risque de renouer avec une vision anthropocentrée et instrumentale réduisant les techniques à des moyens dont seules les sciences humaines pourraient discuter les fins ${ }^{54}$. On retrouve cette idée chez les promoteurs de l'humanisme technique qui

48. Marcel Mauss, Techniques, technologie et civilisation, édition et présentation par Nathan Schlanger, Paris, PUF, 2012.

49. Georges Haudricourt, La technologie science humaine. Recherche d'histoire et d'ethnologie des techniques, Paris, MSH, 1987.

50. Voir par exemple François Sigaut, «Préface, Haudricourt et la technologie», in G. Haudricourt, op. cit, 1987, p. 9-32 ou François Sigaut, "Aperçu sur l'histoire de la technologie en tant que science humaine ", Actes et communications, séminaire INRA CNRS PIRTTEM, histoire des techniques et compréhension de l'innovation, $\mathrm{n}^{\circ}$ 6, 1991, p. 67-82.

51. F. Sigaut, art. cit., 1991, p. 67.

52. Georges Haudricourt, «La technologie, science humaine ", La Pensée, n 115, 1964, p. 28-35. Republié dans Georges Haudricourt, op. cit., 1987, p. 37-46, p. 38.

53. Haudricourt différencie son travail de celui d'André Leroi-Gourhan, également élève de Mauss, qui, pour lui, «s'est intéressé à l'outil, mais n'a pas vu l'homme dans l'outil » au motif que ce dernier était " un bricoleur " (sous entendu : un " techniciste »). "Pour moi [...] le bonhomme est essentiel, c'est le moteur humain ». Entretien, Libération, Bensimon, 1990. Cité par Jean-François BERT, «De M. Mauss à A. G. Haudricourt. Retour sur la technologie ", Revue d'histoire des sciences humaines, $\mathrm{n}^{\circ} 20,2009$, p. 163-181, p. 179.

54. Précisons toutefois que les études de terrain de la " technologie culturelle " sont bien plus riches que ne le laissent penser les écrits qui théorisent leur conception de la technologie. Voir par exemple A. G. Haudricourt, "Domestication des animaux, culture des plantes et traitement d'autrui ", L'Homme, n 2, 1962, p. 40-50, ou F. Sigaut, "Les conditions d'apparition de la charrue. Contribution à l'étude des techniques de travail du sol dans les anciens systèmes de culture ", Journal d'agriculture tropicale et de botanique appliquée, vol. 19, n 10-11, 1972, p. 442-478. 
souhaitent "humaniser la technique ${ }^{55}$, comme si celle-ci n'était pas déjà humaine! En voulant l'humaniser, ils la déshumanisent. Pour nous, vouloir définir la technologie comme science humaine à l'exclusion des autres sciences relève d'une posture de surplomb adoptée par repli. Elle est le symétrique inverse de la posture des sciences et techniques de l'ingénieur qui, en vertu du schéma applicationniste, n’auraient pas à tenir compte du social. Ainsi, chacun tire la couverture à soi.

Mais il est tout aussi problématique de vouloir désigner la technologie comme une science tout court. Pour Sigaut, il ne fait aucun doute que la technologie est " la " science de la technique. Selon lui, sa pratique est fondée, a produit des résultats et continue de le faire ${ }^{56}$; la mise en science des techniques ne lui paraît pas en soi problématique. Si la technologie s'est peu développée et n'est pas devenue une discipline scientifique comme les autres, c'est seulement, selon Sigaut, à cause de facteurs exogènes, sociaux et culturels. Pour lui, ce n'est pas la spécificité épistémique des techniques et du savoir technique qui est fondamentalement en cause, car il en va des techniques comme des autres créations humaines, comme le langage et les institutions : rien ne s'oppose à ce qu'on les étudie pour elles-mêmes sans forcément vouloir les normer ou les améliorer. La faute revient à la « collectivité intellectuelle » qui, considérant les techniques comme de simples moyens, indignes d'intérêt, n'accepte pas que le savoir technique ait en soi une valeur culturelle et intellectuelle ; il ne pourrait avoir qu'un but utilitaire, subordonné à des fins socio-économiques. Pour Sigaut, la valeur économique ou pratique qu'on a pu assigner aux travaux de technologie est inessentielle ; elle relève d'un malentendu qui doit être dissipé ; la technologie n'accédera à une véritable existence disciplinaire qu’à condition de se voir reconnaître son statut de science désintéressée, autotélique ${ }^{57}$.

55. Par exemple Gaston Berger ou Jean Capelle. Voir Marie Escudié, « Politique de l'esprit chez Gaston Berger ", Cahiers RECITS, n 7, 2010, p. 47-62. CAPELLE Jean, L'école de demain reste à faire, Paris, PUF, 1966, p. 108.

56. François Sigaut, "More (and enough) on technology!", History and technolgy, vol. 2, 1985, p. 115-132, p. 123.

57. «Il faudrait tout simplement accepter le fait que la connaissance des techniques a une valeur culturelle et intellectuelle en tant que telle, qu'il s'agisse de l'art de casser les cailloux chez les australopithèques, de la construction des centrales nucléaires ou de l'intelligence artificielle. Toutes ces techniques sont des productions humaines, des faits sociaux. Elles ont toutes le même intérêt pour qui les considère du point de vue des sciences sociales, mais cette attitude paraît encore étrange et même inconcevable à une grande partie de notre intelligentsia ". Ruth Scheps, François Sigaut, 
Si Sigaut renvoie dos à dos le schéma applicationniste et le "biais théoricien " des sciences humaines et sociales (qui consiste à " ne s'intéresser aux techniques que pour autres choses que ce qu'elles sont ${ }^{58} »$ ), on pourrait reprocher à sa conception de la technologie comme science fondamentale un biais théorétique qui l'affaiblit et l'aveugle : qui l'affaiblit en réinstituant les mêmes partages que ceux qui causèrent l'abandon des anciens projets de technologie (entre science pure et science appliquée, théorie et pratique, science et technique); qui l'aveugle en occultant ses conditions de constitution et en dispensant d'une réflexion sur les finalités normatives motivant les différents projets de technologie.

En effet, Sigaut n'hésite pas à faire le pont et à rattacher la technologie culturelle à la technologie beckmannienne, quand bien même Mauss ne fait jamais mention - à notre connaissance - des travaux de Beckmann. Il est vrai que chez Beckmann comme chez Sigaut la technologie apparaît comme une science de la production humaine. Pour autant, les finalités de ces deux technologies sont bien différentes. Pour Sigaut, "nous avons besoin de connaître les techniques pour un ensemble de raisons que nous pouvons qualifier de culturelles, et indépendamment de toutes considérations d'ordre utilitaire ${ }^{59}$ ". L'objectif de la technologie beckmannienne est tout autre : elle ne s'adresse certes pas directement aux artisans et praticiens $s^{60}$, mais plutôt aux futurs administrateurs en charge d'assurer et d'organiser le développement économique du territoire dans une volonté étatique de maîtrise des procédés de fabrication artisanaux ${ }^{61}$.

"La technologie, une science humaine, entretien avec F. Sigaut », in Ruth Scheps (dir.), L'empire des techniques, Paris, Seuil, 1994, p. 51-61.

58. F. Sigaut, art. cit., 1991, p. 71.

59. F. Sigaut, art. cit., 1987, p. 11. Voir aussi F. Sigaut, art. cit., 1991, p. 67.

60. Dans l'Entwurf, Beckmann change de position et conçoit que son ouvrage puisse aussi servir aux savants et praticiens à perfectionner les inventions. G. GARnER, "Johann Beckmann et les sciences camérales du XviII e siècle ", in G. Carnino, L. Hilaire-Pérez, J. Hoock, op. cit., p. 123138, p. 132 et p. 137. Il ne faut pas pour autant négliger la dimension de dépossession que recouvre la formalisation des savoir-faire des artisans, qui relève d'une " désincorporation " non seulement au sens d'abolition des corporations et de la recomposition des métiers dans le paysage révolutionnaire décrit par Alain Cottereau, mais également au sens d'un transfert par lequel les savoirfaire incorporés par les artisans se trouvent comme encapsulés dans des écrits et des dessins. Alain Cottereau, "La désincorporation des métiers et leur transformation en "publics intermédiaires". Lyon et Elbeuf, 1790-1815", in Steven L. Kaplan, Philippe Minard (dir.) La France, malade du corporatisme? XVIII'-XX' siècles, Paris, Belin, 2004, p. 97-145. Alain Cottereau emprunte lui-même l'expression " désincorporation " à Claude Lefort.

61. G. Carnino, L. Hilaire-Pérez, art. cit., p. 22. 
Les articles accompagnant la récente traduction française de l'Entwurf permettent d'ailleurs de relativiser la revendication de la technologie à être une "science autonome ${ }^{62}$ et de contextualiser sa place dans la trajectoire de Beckmann, dont, nous dit-on, il ne faut pas «surestimer la cohérence intellectuelle ${ }^{63} »$. Elle résulterait de la concurrence entre professeurs de Göttingen qui entraîne une forte spécialisation et l'ouverture de nouveaux créneaux disciplinaires exigeant l'écriture de manuels, en l'occurrence l'Anleitung. Il existe également une concurrence interuniversitaire conduisant Göttingen à privilégier l'enseignement en vogue des sciences camérales, dont celui de Beckmann en technologie, qui rencontre un engouement auprès de l'élite de la jeunesse destinée à des postes d'encadrement ${ }^{64}$. In fine, Sigaut décontextualise la technologie beckmannienne en isolant son contenu scientifique de sa normativité pour la transmuer en une science désintéressée qu'il peut dès lors lier à l'effort de la technologie culturelle. Ce faisant, il procède à une réécriture de l'histoire. Il développe une lecture transhistorique de la technologie comme science. Celle-ci se manifesterait à travers les époques sous la forme d'un intérêt épisodique pour les techniques sans parvenir à se maintenir. Par-delà ses interruptions, il y voit la permanence cyclique d'un même motif ${ }^{65}$. Cette hypothèse présuppose une continuité retrouvée. Elle associe un fond théorique commun resté inexploité à des projets de technologie tous avortés. Elle aplanit les différentes normativités propres à chaque projet de technologie. Car les technologies

62. Hélène VÉRIN, art. cit.

63. Guillaume Garner, art. cit., p. 125. Andre WaKefield, "Beckmann in Göttingen ", in G. Carnino, L. Hilaire-Pérez, J. Hoock, op. cit., p. 113-121. On perçoit déjà dans les travaux d'histoire de la technologie précédents une certaine mise à distance de cette cohérence reconstruite par Beckmann lui-même : "on pourrait dire que le mûrissement du projet de Beckmann tel qu'il nous le présente au début de son ouvrage a tous les traits d'un mythe fondateur. La narration de la découverte, par le héros, de l'essence de la technologie, qui surgit de la rencontre des expériences, à l'épreuve renouvelée de la redoutable diversité des arts et des métiers constitués. C'est dans la tête de ce héros-là que s'est imposée comme une révélation la catégorie fondatrice d' "intention" sous laquelle sont venus se ranger les moyens de l'intention ». H. VérIN, art. cit., p. 138.

64. A. WAKEFIELD, art. cit., p. 119.

65. F. Sigaut énumère plusieurs de ces épisodes : celui où il écrit, dans les années 1980 qui inaugure une période de redécouverte du thème de la culture technique. Il situe l'épisode précédent dans les années 1930 où se développent la technologie culturelle et l'histoire des techniques dans les Annales. Ensuite, dans les années 1875-1880 marquées par les travaux de Franz Reuleaux en Allemagne, Pitt-Rivers en Angleterre ou Alfred Espinas en France. Puis il revient à la technologie allemande et à l'Encyclopédie au tournant du XIX ${ }^{\mathrm{e}}$ siècle, en précisant qu'il serait possible de remonter plus loin dans l'histoire. F. Sigaut, art. cit., 1991, p. 72. 
ne proposent pas qu'un savoir de l'action mais aussi, indissociablement, un savoir en action.

\section{Desserrer le nœud Beckmann}

Le moment Beckmannien est généralement considéré comme le point nodal de la technologie, voire son moment paradigmatique. Pour nous, il conviendrait de desserrer ce nœud, sinon de le dénouer, pour replacer la technologie dans des horizons de pluralisation et de relative indétermination sémantiques qui, loin d'en miner la signification, en ouvrent les possibles.

L'indétermination première de la technologie réside dans sa construction sémantique : associant les termes grecs technè (art au sens contemporain de technique) et logos (discours, raison), elle est à la fois art du discours (technè du logos) et discours sur les arts (logos de la technè). Ces deux sens, qui bornent le spectre définitionnel que parcourt la technologie, renvoient l'un à l'autre et se rejoignent par redoublement. Lorsque l'art du discours est formalisé il devient discours sur cet art et lorsque le discours sur les arts est ordonné rhétoriquement il devient art du discours. En effet, la technologia antique, que l'on doit notamment à Cicéron, s'apparente à l'art rhétorique mais dès lors qu'elle s'approche d'une classification ordonnée elle peut aussi être considérée comme un discours sur les arts ${ }^{66}$.

Dans son sens d'art du discours, la technologie a tendance à être connotée péjorativement $^{67}$, mais ce n'est pas toujours le cas. Au XVII ${ }^{\mathrm{e}}$ siècle, la

66. G. Carnino, L. Hilaire-Pérez, art. cit., p. 16.

67. Le Dictionnaire universel de la langue française de Pierre-Claude-Victor Borste (Paris, 1800) la définit comme "fausse science des faux mots " (cité par J. Guillerme, art. cit., 1984, p. 17). Guillerme cite d'autres propos du XIX $x^{e}$ siècle où la technologie est affublée des épithètes «lourde ", « épouvantable ", « superflue ». C'est encore l'aspect livresque d'une technologie éprise de classification et de terminologie qui pousse Gérard Joseph Christian, le premier directeur de Conservatoire national des arts et métiers, à choisir un autre terme pour désigner son grand projet de réforme de l'industrie et de la société : la " technonomie " (1819). C'est enfin cette connotation péjorative que l'on retrouve jusque dans les Universités de technologie, où certains chercheurs déplorent, depuis un point de vue d'ingénieur, qu'on leur demande de "produire du papier " (de la littérature académique, du verbe...). Voir Sacha Loeve, Timothée Deldicque, Pierre Steiner, "Éléments d'anthropologie des sciences humaines et sociales en univers technologique ", Cabiers Costech, n ${ }^{\circ} 1$, 2017, p. 42, p. 51, et p. 100-101. Ces usages péjoratifs, où " technologie " connote une sophistication verbeuse, pédante et toute académique, prennent racine dans le bas latin techna. De techna 
technologie est une "logique des arts ", chapeautée par une métaphysique dont elle est partie intégrante ${ }^{68}$. Elle s'attache aux divisions et aux classifications générales des arts et des sciences dans une optique d'eupraxia, ou norme du «bien-faire ", alliant étroitement idéaux d'élévation spirituelle et visées de classification, de juste délimitation des arts. Dans le Metaphysicae systema methodicum de Clemens Timpler (1604), la technologie est le " traité le plus général et le plus utile qui soit de la nature et de la division des arts libéraux ", classant les disciplines selon la méthode ramiste des dichotomies ${ }^{69}$. Si elle ne s'intéresse pas aux arts mécaniques, leur inclusion s'effectue avec les prologues du mouvement encyclopédique. Dans la Technometria de William Ames (1629), définie comme " la connaissance préliminaire de tout art, qui circonscrit convenablement les limites et la finalité de chacun et de tous les arts ", il est dit que " la division communément admise de l'art en théorique et en pratique est défectueuse à bien des égards et doit donc être rejetée ». Dans l'Encyclopaedia de Johann Heinrich Alsted, en sept tomes et trente-cinq livres (1630), la définition de technologia reste à peu près la même, "doctrine provisionnelle de l'affectation, de l'ordre et de la répartition des disciplines ", mais les disciplines prises en compte relèvent des arts libéraux comme des arts mécaniques (de la gymnastique à l'alchimie, en passant par l'usage du tabac). Au sein du mouvement encyclopédique du XVII ${ }^{\mathrm{e}}$ siècle, la technologia est donc une métadiscipline chargée de dessiner la " carte des savoirs ». Chez Alsted, elle fait face à ontologia, qui relie chaque discipline de l'encyclopédie à son domaine d'objet délimité, tandis que technologia organise les relations des disciplines entre elles, épaulée en cela par hexiologia (doctrine de l'exercice des habitudes intellectuelles), archeologia (doctrine des origines, des principes et des normes de chaque discipline) et didactica. La technologie n'y est pas un savoir appliqué aux pratiques matérielles, mais la forme donnée à l'encyclopédie, c'est-à-dire à la mise en cercle des savoirs dans une optique de paideia (éducation-élévation), pour que son système intègre efficacement un maximum d'activités humaines et les élèves à la dimension du logos. Il s'agit de faire entrer les arts mécaniques comme les autres arts dans la

dérive aussi le terme aujourd'hui désuet de " technie ", pouvant signifier partie pratique d'un art, mais aussi sophistication verbeuse, jargon (les " termes techniques").

68. C'est ainsi qu'en 1653, l'appellation theses technologicae remplace celle de theses metaphysicae à Yale et Harvard jusque dans les dernières décennies du XviII siècle. Porter G. Perrin, "Possible sources of technologia at early Harvard", The New England Quaterly, vol. 7, n 4, 1934, p. 718-724. 69. Sur la technologia de Pierre de la Ramée, voir les travaux de Koen Vermeir. 
sphère du logos, mais c'est la forme qui est technologique, non le contenu. Il ne s'agit pas de tirer un logos des arts mécaniques eux-mêmes, comme plus tard chez Beckmann.

Cependant, on aurait tort selon nous de cantonner l'ancienne technologia encyclopédiste à une sorte de " préhistoire » de la technologie, comme si sa véritable histoire commençait avec Beckmann (après de premières lueurs chez Wolff). Non seulement cette "préhistoire " mérite d'être pleinement réintégrée dans l'histoire de la technologie, mais cette histoire à son tour, gagnerait à être plus franchement délinéarisée. La périodisation suggérée par Guillerme dans "Les liens du sens $»^{70}$, qui décrit le passage d'un régime rhétorique de la technologie à un régime métaphysique, puis à un régime artisanal et enfin industriel, est encore trop linéaire. Si la distinction entre ces grands régimes est utile, ils présentent moins des rapports de succession que de coexistence ${ }^{71}$. Même l'Entwurf maintient et assume une ambition nomenclatrice qui le place au sein du registre des ouvrages de terminologie $^{72}$. Cet aspect, outre qu'il nuance l'idée d'une rupture avec l'ancienne technologia, explique en partie l'efficacité performative de la tentative de Beckmann dans la mesure où «l'entrée en technologie par l'apprentissage se fait [...] par le truchement des termes, véritables vecteurs non seulement de connaissances, mais aussi de pratiques ${ }^{73}$ ". Ainsi le sens ancien de technologia comme logique des arts ne nous semble aucunement relégué à un passé dépassé. Ce qu’on appelle l'ingénierie des connaissances aujourd'hui, par exemple, en relève pleinement. Ou encore, l'ancienne technologia n'est pas sans évoquer un des sens que prend la technologie dans les premiers textes de Gilbert Simondon : non pas une discipline particulière qui s'intéresserait aux artefacts techniques mais une méthodologie interscientifique. Selon lui, si chaque science est définie par un rapport entre la connaissance et une structure du réel délimitant une ontologie régionale, la technologie échappe à ce schéma sujet-objet : elle est tout autant entre les sciences qu'entre les domaines techniques. Elle n'est pas

70. J. Guillerme, art. cit., 1984.

71. Certes Guillerme ne parle pas de succession simple, où un nouveau régime remplace l'ancien, mais d'une « longue suite de catachrèses où se conservent, néanmoins, des rapports d'analogie entre ses objets successifs de référence ». Ibid., p. 18.

72. Danielle Candel, John Humbley, "Lémergence d'une conscience terminologique : regard métalexicographique porté sur l'Entwurf der algemeinen technologie de Johann Beckmann (1806) ", in G. Carnino, L. Hilaire-Pérez, J. Hoock, op. cit., p. 199-219.

73. Ibid., p. 219. 
assignée à une ontologie régionale mais s’attache aux régimes de fonctionnement permettant de passer d'un domaine à un autre via les analogies d'opération entre ces régimes ${ }^{74}$.

L'historiographie de la technologie a maintes fois mis en avant la rupture avec l'ordre descriptif de l'Encyclopédie de Diderot, d'Alembert et al. que la technologie beckmanienne aurait accomplie en passant d'une classification par filières et métiers à une classification par opérations analogues. Or cette " rupture épistémologique » mérite aussi d'être questionnée.

D'abord, l'idée d'analogie entre des opérations communes à divers métiers n'est pas absente de l'Encyclopédie. La classification ne s'y fait pas par métiers uniquement, mais par matières premières transformées par les métiers. Or, entre matières et métiers, il y a les outils, et malgré le flottement des termes les désignant déploré par Diderot, ceux-ci sont souvent vecteurs d'analogies d'opération, faisant communiquer matières et métiers via le réseau des renvois de l'Encyclopédie $e^{75}$.

Mais surtout, l'usage que Beckmann fait de l'analogie d'opération est ambigu et, à y regarder de plus près, loin d'être épistémologiquement robuste. Il écrit : les " métiers, aussi différents que soient leurs matériaux

74. Gilbert Simondon, "Allagmatique " (1953), in L’individuation à la lumière des notions de forme et d'information, Grenoble, Millon, 2005, p. 559-566 ; "Cybernétique et philosophie " (1953), in Sur la philosophie, Paris, PUF, 2016, p. 35-68, p. 42-43. Voir aussi Xavier Guchet, Pour un humanisme technologique. Culture, technique et société dans la philosophie de Gilbert Simondon, Paris, PUF, 2018 : "la technologie, non pas science des machines seulement, mais science générale des opérations dans l'être ", p. 16.

75. Par exemple, le "polissoir " est un outil qui renvoie à différentes opérations (" polir ", " gratter ", " tanner »...) distribuées dans différents métiers (" aiguillier ", " éperonnier ", " doreur ", " coutelier ", ...) ; chacune des opérations dévoile à son tour une constellation d'outils, de matières et de métiers : "gratter ", renvoie au "batteur d'or ", au "formier ", au " menuisier ", au "graveur ", etc. Dans l'article "Art ", Diderot souhaite " qu'on eût plus d'égard à l'analogie des formes et des usages » des outils, et qu'on détermine « la différence et [...] la ressemblance des formes et des usages d'un instrument et d'un autre instrument, d'une manœuvre et d'une autre manœuvre, quand il faudrait leur laisser un même nom et leur donner des noms différents ". Dans L'histoire et le secret de la peinture en cire (1755), il esquisse une théorie de l'invention visant à découvrir les « rapports utiles et ignorés " entre les arts et à inciter les artisans à se prêter " un secours mutuel ", allant dans le sens du décloisonnement des métiers. Voir Sophie De Beaune, Liliane Hilaire-Pérez, Koen Vermeir, "L'analogie, les techniques et les savoirs. Approches cognitives et historiques", in S. De Beaune, L. Hilaire-Pérez, K. Vermeir, op. cit., p. 7-56, p. 37-38. Voir aussi Antoine Picon, "Gestes ouvriers, opérations et processus techniques. La vision du travail des encyclopédistes ", Recherches sur Diderot et l'Encyclopédie, n 13, 1992, p. 131-147. 
et leurs produits, ont à exécuter des opérations $\left(\right.$ Arbeiten $\left.^{76}\right)$ diverses en vue d'un même but (Absicht) ; ou savent atteindre le même but de façons très différentes ${ }^{77}$ ". Sa technologie générale se donne pour tâche de cataloguer ces différents buts ou intentions constitués en classes vedettes puis de ranger dans chacune d'elles toutes les tâches ou opérations particulières s'y rapportant. Beckmann liste vingt-trois classes mais en traite seulement deux : lisser et fragmenter, regroupant respectivement dix-neuf et trente et une opérations ${ }^{78}$. La manière dont Beckmann souhaite construire les articles de la technologie générale - une partie générale consacrée au but commun et une partie spécifique traitant des " moyens " de réaliser ce but $^{79}$ - est caractéristique d'une vision instrumentale des techniques où les moyens - les opérations ou tâches (Arbeiten) - sont subordonnés à des fins - les intentions - qui leur sont extérieures et les dépassent.

Cependant, il y a selon nous chez Beckmann une indissociation entre intention et opération, également remarquée par Vincent Bontems qui pointe " une certaine indistinction entre analogies opérationnelles et analogies fonctionnelles chez Beckmann ${ }^{80}$ ». Si on s'en tient à ce que dit Beckmann, l'analogie concerne la fonction, c'est-à-dire l'intention visée par l'opération ; elle permet de classer les opérations mais ne porte pas directement sur elles ; les opérations d'une classe ne sont pas les cas particuliers d'une opération générique mais d'une fonction. C'est pourquoi Joost Mertens peut parler d'une " analogie "éloignée" » entre, par exemple, les opérations " briser " et " arroser ", qui ont un but analogue, "fragmenter ", mais n'ont

\footnotetext{
76. Littéralement, des "travaux " ou " tâches ". Dans son "Avant-propos " à la traduction de l'Entwurf, Joost Mertens précise : «j'ai rendu presque invariablement Arbeiten (le substantif, au pluriel) par opérations. Ce terme plus récent exprime parfaitement, je crois, la pensée de Beckmann. Je voulais aussi faire ressortir le fait que sa technologie est opératoire, c'est-à-dire une théorie dont les objets centraux sont les opérations, pas les métiers ni les marchandises ", in G. Carnino, L. Hilaire-Pérez, J. Ноock, op. cit., p. 10.

77. J. Beckmann, Projet de technologie générale, op. cit., p. 67-68.

78. J. Beckmann, Projet de technologie générale, op. cit., p. 67-68, p. 76-102. Voir Joost Mertens, "L'analogie : principe structurant de la technologie générale, principe dynamique du transfert industriel ", in S. De Beaune, L. Hilaire-Pérez, K. Vermeir, op. cit., p. 141-160, p. 151-152. «1. Lisser ; 2. Rendre rugueux ; 3. Fragmenter ; 4. Mouiller ; 5 . Sécher ; 6. Redresser ; 7. Plier ; 8. Durcir ; 9. Raidir ; 10. Condenser ; 11. Désagréger ; 12. Amincir ; 13. Tamiser ; 14. Réchauffer ; 15. Refroidir ; 16. Rendre transparent ; 17. Rendre opaque ; 18. Rendre élastique ; 19. Rendre flexible (les corps liquides) ; 21. Décolorer ; 22. Faire évaporer ; 23. Rendre souple».

79. J. Beckmann, Projet de technologie générale, op. cit., p. 74.

80. Vincent Bontems, "Analogies techniques et raisonnements analogiques (une lecture simondonienne) », in S. De Beaune, L. Hilaire-Pérez, K. Vermeir, op. cit., p. 273-289, p. 285.
} 
que peu de choses à voir en termes de schèmes opératoires ${ }^{81}$. Toutefois, l'effort de Beckmann consiste à " assimiler l'acte opératoire à une intention " et à se tenir " au plus près de l'exercice de l'art " en désignant les intentions comme les opérations non par des substantifs mais par des verbes d'action ${ }^{82}$. Ainsi, dans leur formulation même, opérations et intentions tendent à se superposer, à coïncider et à se confondre. V. Bontems écrit : " "lisser" semble au premier abord une opération alors qu'il s'agit plutôt d'une fonction [ici au sens d'intention] que plusieurs opérations réalisent ${ }^{83} »$.

Cette confusion est rendue possible par une relative interchangeabilité des fins et des moyens dans la vision instrumentale de Beckmann. Parce qu'elle se tient au plus près de l'opération pour la décrire exactement, l'intention beckmannienne ne s'identifie pas forcément à une finalité générale, mais aussi, bien souvent, à un moyen pour autre chose. Si on lisse pour quelque chose d'autre, "lisser " n'est qu'une étape ; "lisser " s'insère en tant qu'intention d'une ou plusieurs opérations dans une concaténation ou une chaîne d'opérations dont la finalité est plus générale. La notion beckmannienne d'intention se situe à mi-chemin entre celles d'opération et de finalité. Elle n'est déjà plus l'opération et ne peut pas l'être car elle entretient avec elle un rapport discursif mais elle ne désigne pas encore la finalité ou l'utilité générale d'une ou plusieurs opérations. La tension non résolue entre intention et opération chez Beckmann montre l'instabilité du sol sur lequel on voudrait fonder la technologie.

Cette tension est révélatrice du niveau élevé de granularité de l'analyse opératoire entreprise par Beckmann. Comme le souligne Hélène Vérin, la décomposition analytique des arts et métiers en opérations ou tâches (Arbeiten) qu'on trouve chez Beckmann est à rattacher aux réflexions sur la division du travail initiées dans la seconde moitié du XvıII ${ }^{\mathrm{e}}$ siècle. Selon Antoine Picon, cette "rationalité analytique " travaille déjà en creux la classification descriptive des arts et métiers de l'Encyclopédie où « la notion d'opération occupe une place centrale [...] comme une sorte d'unité

81. J. Mertens, art. cit., p. 152-153. V. Bontems écrit également que «Beckmann souligne luimême la disjonction entre différents aspects de la technique quand il formule des analogies plus lointaines, par exemple, les actions "briser" et "arroser", qui sont fonctionnement analogues mais doivent recourir à des opérations distinctes selon les matériaux ». V. Bontems, art. cit., p. 285.

82. Comme le souligne H. VÉRIN, art. cit., p. 139.

83. V. Bontems, art. cit., p. 285. 
sémantique de base qui permet de passer du savoir-faire individuel à la logique d'ensemble d'une production " et participe de l'émergence de la notion de travail humain comme facteur essentiel de la production ${ }^{84}$. Au XIX siècle, Gérard-Joseph Christian en France puis Charles Babbage et Andrew Ure en Angleterre poursuivent cette démarche ${ }^{85}$. La décomposition analytique des arts et métiers en opérations permet de quantifier le travail humain, d'améliorer la production et de la mécaniser en faisant réaliser certaines opérations, sinon toutes, par des machines. Marx critique cette abstraction du travail humain dont il fait le propre de « cette science toute moderne qu'est la technologie ${ }^{86} »$. Cependant, ces conceptions et projets technologiques ont des formes, des intentions et des contextes de déploiement tous très différents. Ils sont irréductibles les uns aux autres et possèdent leur normativité propre, qu'il n'est pas légitime de gommer au profit d'une lecture transhistorique de la technologie où Beckmann tiendrait le premier rôle.

84. A. Picon, art. cit., p. 144-145.

85. Gérard-Joseph Christian, Vues sur le système général des opérations industrielles, ou Plan de technonomie (1819) ; Charles BABBAGE, On the economy of machinery and manufactures (1832); Andrew Ure, Philosophy of manufactures (1835). Voir Joost Mertens, "The mere handicrafts: Ure's Dictionary (1839-1853) compared with the Dictionnaire technologique (1822-1835)”, Documents pour l'histoire des techniques, vol. 19, 2010, p. 277-285.

86. Karl Marx, Le capital. op. cit, p. 546. Notons que les piques que Marx lance à la technologie n'excluent pas des jugements plus positifs voire laudatifs : "la technologie a [...] découvert les quelques grandes formes fondamentales du mouvement selon lesquelles, malgré toute la variété des instruments utilisés, toute action productive du corps humain procède nécessairement, tout comme la plus grande complexité des machines ne trompe pas la science mécanique sur la répétition perpétuelle de puissances mécaniques simples. L'industrie moderne ne considère et ne traite jamais la forme actuelle d'un procès de production comme si elle était définitive. C'est pourquoi sa base technique est révolutionnaire. » (Ibid., p. 547). Ou encore : «Darwin a attiré l'attention sur l'histoire de la technologie naturelle, c'est-à-dire sur la formation des organes des plantes et des animaux en tant qu'instruments de production de la vie [...]. Mais l'histoire de la formation des organes productifs de l'homme social, de la base matérielle de toute organisation particulière de la société, ne mérite-t-elle pas la même attention ? [...] La technologie révèle le comportement actif de l'homme envers la nature, le procès immédiat de production de sa vie, donc aussi des conditions sociales de son existence et des conceptions intellectuelles qui en découlent » (p. 417-418, note 89). Et Marx de prophétiser qu'outre " l'enseignement élémentaire [première concession arrachée de haute lutte au capital], il ne fait pas le moindre doute que, grâce à l'inéluctable conquête du pouvoir politique par la classe ouvrière, l'enseignement de la technologie, pratique et théorique, conquerra lui aussi sa place dans les écoles du peuple » (p. 548-549). Dans ces lignes, il oppose l'enseignement de la technologie à la formation professionnelle par filières, qu'il dépeint comme une stratégie d'optimisation du travail par le capital. D’une certaine manière, l'enseignement d'une technologie non spécialisée rencontre l'idéal de l'individu émancipé que défend Marx, celui d'une vie polyvalente, consacrée à des activités multiples : chasseur le matin, éleveur l'après-midi, critique littéraire le soir... 
En résumé, les sens de technologie ne se succèdent pas les uns aux autres mais les différentes technologies coexistent, ce qui explique la discordance des acceptions du terme, y compris à une même époque donnée. Un historien des sciences kuhnien pourrait dire de la technologie qu'elle n'a jamais dépassé son stade préparadigmatique ; qu'elle n'est jamais devenue une "science normale » comme la physique newtonienne, la chimie organique, la biologie moléculaire ou l'économie néoclassique, chacune procédant de la constitution et de la consolidation d'un paradigme stable avec des idéalisations partagées rendant possible une croissance cumulative des connaissances sur le temps long. Les différents projets de technologie se sont très peu influencés et ont très peu hérité les uns des autres ; ils n’ont pas donné lieu à une capitalisation des connaissances. La technologie n'a jamais fait paradigme, même pendant la période caméraliste. Mais cela ne signifie pas qu'elle doive nécessairement viser à le faire, bien au contraire.

\section{La remotivation du projet d'université de technologie (UT)}

Le nœud beckmannien maintenant desserré, nous voudrions attirer l'attention sur la spécificité d'un projet de technologie plus récent. Créée en 1972, l'université de technologie de Compiègne (UTC) opère une remotivation du concept de technologie en jouant sur la multiplicité de ses sens anciens et contemporains. Guy Deniélou, issu du Commissariat à l'énergie atomique (CEA), fondateur et premier président emblématique de l'UTC, connaît et reprend à son compte la signification ancienne de technologie. Deniélou écrit : "l'université de Compiègne propose "sa" définition officielle de la technologie : "c'est le nom de la science quand elle prend pour objet les produits ou les procédés de l'industrie humaine" ». Dans le même texte, il fait directement référence à Beckmann en ces termes : " comment ne pas évoquer la mémoire de Beckmann qui, à Göttingen, en 1777, a peut-être le premier fait usage du mot même technologie ? ». Il déplore l'absence de traduction française de ses ouvrages ${ }^{87}$. Beckmann apparaît comme une illustre figure tutélaire, idéale pour légitimer le projet UT, qui

87. Guy DeniéLou, "Technologie ", La grande encyclopédie alpha des sciences et des techniques. Technologie I, $\mathrm{n}^{\circ}$ 166, 10 mai 1977. Republié dans Guy DeniéLou, Propos sur la technologie. Fragments (1972-1986), édition numérique non publiée établie en 2011 par Jean-Pierre DHOURY, p. 23. 
s'inscrit lui-même dans un processus contemporain de disciplinarisation de la technologie à tous les niveaux scolaires. Le modèle UT met également à profit le sens contemporain de technologie qui fait écho aux valeurs de scientificité, d'innovation et de modernité. Son modèle de référence, constamment invoqué dans la rhétorique UT dès ses débuts, est le MIT ${ }^{88}$. L'impression donnée est que le mot technologie fait figure d'attrape-tout, qui, en suscitant l'intérêt du secteur industriel et des décideurs politiques, a permis au modèle UT d'exister. Pour autant, si l'identité de l'UTC se compose à la fois du sens ancien et du sens contemporain de technologie, elle ne se réduit à aucune de ces deux significations.

À la fois école d'ingénieurs et université, le modèle UT représente une troisième voie entre ingénierie et académie construite sur la notion de " technologie ", choix indissociablement épistémologique et institutionnel fait en lieu et place de celui du couple sciences fondamentales/sciences appliquées $^{89}$. "Le fait de l'afficher dans le titre indique que l'on veut combler un fossé, celui qui sépare l'Université de l'industrie ${ }^{90} »$. Dans ses écrits, G. Deniélou, énonce les caractéristiques épistémologiques et organisationnelles d'un tel modèle. Il consiste à croiser les approches des « logies " (les disciplines académiques en sciences de la nature et de l'humain) et des " génies" (secteurs industriels) et s'oppose en cela à toute hiérarchie ou linéarité entre le "fondamental " et "l'appliqué ». Dans ce cas la technologie n'est pas une "logie " particulière (celle des techniques) mais ce couplage à géométrie variable entre les « logies » et les " génies " produisant à la fois de nouvelles méthodologies de conception, de nouveaux procédés et produits industriels, et de nouvelles connaissances. Ainsi, G. Deniélou écrit : la technologie "désigne parfois, seulement dans certains cercles, cette logie particulière qui est l'étude de la tekhnê. Mais, le plus souvent,

88. Pierre Lamard, Yves-Claude Lequin, La technologie entre à l'université. Compiègne, Sevenans, Belfort-Montbéliard..., Belfort-Montbéliard, UTBM, 2005, p. 94. Il faut toutefois noter le paradoxe puisque le technology du MIT fait référence au sens ancien de technologie.

89. "Le pôle principal choisi est la Technologie. [...] Il régnait plus ou moins confusément dans l'Université l'opinion que la physique suffisait à fonder la technologie. Dans les milieux industriels, au contraire, prévalait l'impression que les exigences propres aux marchés et à la fabrication l'emportaient sur tous les autres. Des deux côtés semble-t-il, on se refusait à reconnaitre l'irréductibilité du technique et l'émergence d'une nouvelle discipline. Guy DeniéLou, « Pourquoi créer une université à Compiègne ?", Revue d'information de l'Oise, 1972. Reproduit in G. DeniÉLou, op. cit., p. $72-74$, p. 72.

90. Fond UTC, Compte-rendu du symposium des 16 et 17 septembre de Bellinglise, p. 2. Cité par Pierre Lamard, Yves-Claude Lequin, op. cit., p. 95. 
elle évoque cette approche à la fois théorique et pratique, en tout cas scientifique, de nos produits et de nos procédés sur un mode à la fois interdisciplinaire et interprofessionnel ${ }^{91} »$.

Dans cette technologie interdisciplinaire qui rejette l'applicationnisme, la place des sciences humaines et sociales (SHS) est revalorisée. G. Deniélou a l'ambition de former des "ingénieurs-philosophes " ${ }^{92}$. Au départ, les SHS sont intégrées à la formation de l'ingénieur par l'intermédiaire du design qui joue un rôle d'intercesseur entre SHS et science de l'ingénieur ${ }^{93}$, notamment via un cours de "culture technique " enseigné par Yves Deforge ${ }^{94}$. Les étudiants y sont invités à reconstituer des lignées d'objets techniques selon leur principe de fonctionnement et leur fonction d'usage en analysant le réseau des contraintes techniques et sociales dont ils procèdent. Ce travail d'acquisition d'une culture technique doit leur permettre de dégager une marge de liberté parmi ces contraintes et de l'investir pour penser la conception future de l'objet ${ }^{95}$. Aujourd'hui, les SHS sont constituées en département (Technologie et sciences de l'homme, TSH, 1986) et laboratoire (Connaissance, organisation et systèmes techniques, COSTECH, 1993). Avec les équipes de SHS des autres universités de technologie, Belfort-Montbéliard (UTBM, 1985) et Troyes (UTT, 1994), ils forment un groupement d'intérêt scientifique nommé « Unité des technologies et des sciences de l'homme " (GIS UTSH, 2013) promouvant la " recherche technologique en SHS ». Le GIS UTSH invite, d'une part, les SHS à prendre la mesure du caractère constitutif des techniques pour l'humain et

91. Guy DeniéLou, "Les génies et les logies ", Réforme, mai 1981. Republié in G. DeniéLou, op. cit., p. 84-86.

92. Guy Deniélou cité par Pierre STEIner, "Philosophie, technologie et cognition. États des lieux et perspectives", Intellectica, vol. 1-2, n 53-54, 2010, p. 7-40, p. 8. "Nous essaierons de mettre un terme à la coupure absurde qui s'est instaurée entre les humanités et la science. Ici encore, le choix de la technologie est particulièrement judicieux ». G. DeniéLou, art. cit., 1972, p. 73.

93. En 1981, avec le concours de l'enseignante-chercheure et designeuse Danielle Quarante, l'UTC est la première école d'ingénieurs française à ouvrir une spécialité d'ingénieur en design.

94. Yves Deforge est inspecteur de l'enseignement technique, ayant réalisé une thèse sous la direction de G. Simondon. Euvrant à la mise en place d'une éducation technologique au collège des années 1960 aux années 1990, il est présent aux côtés de G. Deniélou dès la création de l'UTC en 1972. Qualifiant sa pratique de "technologie ", il développe une généalogie des traditions technologiques où Beckmann tient une place importante. Voir par exemple. Yves Deforge, De l'éducation technologique à la culture technique, Paris, ESF, 1993, p. 65.

95. Voir Victor Petit et Timothée Deldicque, "La recherche en design avant la "recherche en design" ", Cahiers Costech, n 1, 2017, 35 p., partie 3, "Génétique des objets industriels et design idéologique (Deforge)». 
les connaissances qu'il produit et donc à instrumenter leur pratique afin de renouveler leurs problématiques, et d'autre part, les sciences de l'ingénieur à faire participer les SHS au processus de conception technique en amont afin d'explorer ensemble les potentialités transformatrices des techniques ${ }^{96}$. Dans cette optique, l'UTC a ouvert, en 2012, le cursus Humanités et technologie (Hutech), mêlant à parts égales SHS et sciences de l'ingénieur, qui constitue une alternative au tronc commun de la formation d'ingénieur. L'objectif d'Hutech est d'outiller conceptuellement les étudiants ingénieurs afin qu'ils puissent faire face à la multiplicité des situations qu'ils rencontreront dans leur future profession ${ }^{97}$.

La spécificité du modèle d'enseignement et de recherche des UT n'empêche pas une grande diversité de manières, pour leurs acteurs (ingénieurs d'études, enseignants-chercheurs en sciences de l'ingénieur ou en SHS, étudiants-ingénieurs, etc.), de se saisir de la notion de technologie et de la remotiver. Elle peut donner lieu à une recherche-étude technologique réfléchissant sur la co-constitution de l'humain et des techniques tout comme à une recherche-action de co-conception de dispositifs, la seconde n'étant pas une "application » de la première. Le projet de recherche HomTech (Sciences de l'homme en univers technologique) ${ }^{98}$ a tenté de donner un aperçu de cette hétérogénéitép9 ${ }^{9}$. S'il nous est impossible de la détailler ici, qu'il nous soit permis de nous appuyer sur cette enquête pour affirmer une fois de plus la fécondité des malentendus que génère la technologie. Que ce soit lors de la création du modèle UT ou aujourd'hui dans les pratiques de ses acteurs, ces malentendus constituent une incitation heuristiquement féconde à la formulation de nouveaux projets de technologie, irréductibles

96. Charles Lenay, Pascal Salembier, Pierre Lamard, Yves-Claude Lequin, Loïc Sauvée, « Pour une recherche technologique en sciences humaines et sociales ", Actes du colloque SHST-UPEC, Créteil, 2013, Les sciences humaines dans les parcours scientifiques et techniques professionnalisants : quelles finalités et quelles modalités pratiques?, SHS Web of Conferences proceedings, vol. 13, 05001, 2014.

97. Guillaume CARnino, «Vers une épistémotechnique », Actes du colloque SHST-UPEC, Créteil, 2013, Les sciences humaines dans les parcours scientifiques et techniques professionnalisants : quelles finalités et quelles modalités pratiques?, SHS Web of Conferences Proceedings, vol. 13, 05002, 2014. 98. Coordonné par Pierre Steiner au COSTECH, UTC, Le projet HomTech (2015-2017) a bénéficié du financement du Conseil régional Hauts-de-France et du Fond européen pour le développement régional (FEDER).

99. Voir Sacha Loeve, Timothée Deldicque, Pierre Steiner, "Éléments d'anthropologie des sciences humaines et sociales en univers technologique ", Cahiers Costech, n 1, 2017, 118 p. 
mais jamais complètement étrangers aux anciens, dans lesquels la signification de cette dernière est constamment remise en jeu.

\section{Pluraliser la technologie}

Ainsi les malentendus de la technologie ne sont pas des incidents de parcours qui arriveraient secondairement à une technologie idiosyncrasique pour la détourner de sa signification première et originelle, mais une caractéristique constitutive et féconde de la technologie. Le projet d'instauration d'un champ du savoir nommé « technologie » n'a jamais fait consensus. La technologie se fait mal entendre. Dès qu'elle existe, elle est mal entendue. Cela a toujours été le cas et le sera encore.

Cette thèse n'est pas sans évoquer celle du philosophe Jean-Claude Beaune dans La technologie introuvable ${ }^{100}$, mais la signification que nous lui donnons est autre. Selon Beaune "la technologie désigne le mouvement interne que possède la technique à s'ériger en fait de langage, à dépasser son aspect conjoncturel et évènementiel pour atteindre une rationalité de structure (qu'elle a bien du mal à exprimer par elle-même, pour des raisons culturelles) qui la met en communication virtuelle avec d'autres techniques (en droit, avec toutes les techniques) et la situe dans le contexte virtuel d'un système général des savoirs et des pouvoirs. Cette définition permet de maintenir le rapport entre sa fonction encyclopédique et sa texture interne ${ }^{101} »$. La technologie est donc pour Beaune la recherche d'une parole adéquate au logos interne et systématique des techniques, qu'on pourrait appeler un "parler les techniques ». Mais Beaune distingue, à la manière structuraliste, la parole (l'expression située) de la langue qui la fonde : « en droit (...), toute technique est par elle-même un ensemble de signes plus ou moins organisé, plus ou moins parlant ${ }^{102} »$. Les techniques "parlent plus ou moins ", cependant "c'est le récepteur qui en juge » et qui, selon sa culture, les traduit, et donc les trahit. Beaune qualifie alors sa propre recherche d' "aporétique ", puisqu'elle consiste en une étude internaliste de l'unité de technologie comme langue des techniques, mais appréhendée par ses inévitables réductions dans des langages externes et des discours

100. Jean-Claude Beaune, La technologie introuvable. Recherche sur la définition et l'unité de la Technologie à partir de quelques modèles du XVIIT et XIX siècles, Paris, Vrin, 1980.

101. J.-C. Beaune, op. cit., 1980, p. 81-82 (italiques de l'auteur).

102. Ibid., p. 82. 
indirects (scientifiques, économiques, technocratiques, ou culturalistes), qui constituent des "technologies secondes". La technologie introuvable raconte alors comment la technologie passe à côté de l'expression adéquate de son objet tout en trahissant par-là la positivité de son existence : " La Technologie unitaire existe mais elle ne parle pas, ou mal (on ne la reçoit pas). [...] À travers tous les discours ambitieux et sûrs d'eux-mêmes et toutes les variétés de l'absence, on distingue "au fond" de ces réductions, la présence mal assurée d'une voix des techniques qui n'accepte pas de collaborer à ses langages forcés. (...). On a fait l'expérience constante d'une absence positive ${ }^{103}$. Il y aurait donc une résistance positive irréductible de la technologie à tous les langages qui tentent de l'exprimer. La recherche de Beaune aboutit à l'idée que l'expression la moins réductrice de la technologie ne relève pas d'une raison connaissante mais d'une raison rêvante... Le reste de son œuvre, centré sur l'imaginaire technique, en témoigne.

Pour nous si le logos de la technologie n'est jamais pur ni exprimable (même en droit) par un sujet de la connaissance logothéorique, ce n'est pas parce qu'il est conditionné par une culture et une histoire particulière - fait au fond banal qui ne pose problème qu'au philosophe épris d'abstraction mais parce qu'il est conditionné par une histoire qui est aussi technique $e^{104}$, ce qui par principe lui interdit de dominer son objet sans immédiatement le nier. Le logos des techniques renvoie inévitablement aux techniques du logos, il est techniquement constitué.

Dès lors, la confusion délibérée entre techniques et technologie(s) est un des sens que peut légitimement prendre la technologie. Penser cette confusion, ce nécessaire malentendu, c'est rendre compte du régime d'interchangeabilité entre le discours-sujet et l'objet-discours qui caractérise la technologie. Si le premier est excorporé dans des écrits et le second incorporé dans des dispositifs. Même le livre, d'un point de vue technologie, est à penser comme un dispositif technique, et comme un dispositif spécifique parmi d'autres possibles pour la transmission-traduction (ou transduction) des savoir-faire.

103. Ibid., p. 11.

104. Beaune le dit (Ibid., p. 83) : «De l'ars probandi à l'ars invendi, la continuité est maintenue. Penser c'est calculer mais c'est aussi créer. Et si l'on calcule à l'aide de machines, on crée par les machines des pratiques et des ordres qui ne se réduisent pas à leurs conditions théoriques mais produisent une nouveauté connaissable ". 
Même quand elle est un " discours sur » les techniques, la technologie ne peut pas faire l'économie d'interroger les opérations et les supports techniques d'inscription constitutifs de son énonciation : livre, mais aussi dessin, enregistrement, site internet, base de données, notice, script ${ }^{105}$, ou encore design (le design est à la fois un discours et une mise en forme technique). C'est ce que postule la théorie du support de Bruno Bachimont ${ }^{106}$, pour laquelle tout objet technique est l'inscription matérielle d'une connaissance et toute connaissance est d'origine technique. Ainsi la technologie ne saurait être une science comme une autre - que ce soit sur le modèle des sciences humaines (compréhensives-normatives) ou des sciences de la nature (théoriques-appliquées) - parce qu’elle redéfinit la connaissance par une épistémologie technique.

Comme dit plus haut, logos de technè et technè du logos se rejoignent par redoublement. Pour autant, il n'y a jamais équivalence entre les deux mais rapports dynamiques d'embrayage et de débrayage de supports à contenus, et de discours à objets. On peut tout aussi bien débrayer sur une technique qu'embrayer sur un discours ${ }^{107}$. "Dire la technique ", écrivent Madeleine Akrich et Dominique Boulier au sujet des modes d'emploi, " c'est passer d'une structuration technique à une structuration linguistique, deux médiations qui ne sont jamais équivalentes. Passer de la manœuvre (observée ou éprouvée) au mode d'emploi, c'est passer de la chronologie des opérations à la chronologie propre au récit. Ces passages sont autant de traductions, qui produisent de l'inadéquation (comme toute mise en langage) et du malentendu (comme dans toute communication) ${ }^{108}$ ". Ils posent la question : " pour transmettre la technique, doit-on la dire ? ", suggérant ainsi qu'une technique qui porte en elle les conditions de sa propre transmission peut très bien être considérée comme une techno-logie. On peut, dès lors,

105. Madeleine Aкrich, «Comment décrire les objets techniques?», Techniques et culture, vol. 9 , 1987, p. 49-64.

106. Bruno BaChimont, Arts et sciences du numérique : ingénierie des connaissances et critique de la raison computationnelle, Mémoire d'habilitation à diriger les recherches, université de technologie de Compiègne, 2004 ; Le sens de la technique : le numérique et le calcul, Paris, Les Belles lettres, 2010. 107. Bruno Latour dans Enquête sur les modes d'existence. Une anthropologie des modernes, Paris, La Découverte, 2012, pense les techniques au moyen du concept de "débrayage ", qui permet de transférer un projet dans la réalité, mais n'envisage pas l'opération inverse, celle de l'embrayage sur un discours ou une formalisation.

108. Madeleine Akrich, Dominique Boulier, «Le mode d'emploi », in Denis Chevallier (dir.), Savoir faire et pouvoir transmettre. Transmission et apprentissage des savoir-faire techniques, Paris, MSH, 1991, p. 113-131, p. 129. 
affirmer qu'il y a technologie non seulement quand des savoir-faire sont formalisés sous forme scripturale, mais aussi quand les modes de transmission, de reproduction et de variation des savoir-faire passent eux-mêmes par la technique (ce qui n'interdit pas de les en extraire à nouveau, pour les expliciter, les théoriser, les critiquer). Peuvent donc être dits « technologiques " des objets (et non seulement des discours) dont les modes d'existence incorporent leurs modes de connaissance. En ce sens, il peut y avoir de « bonnes raisons » de confondre la technologie avec son objet.

Ce n'est peut-être pas un hasard si c'est surtout dans les domaines de la biologie et de l'informatique qu'on parle aujourd'hui de technologie avec des expressions comme « biotechnologies » ou « technologies de l'information et de la communication ». On ne dit pas «biotechnique " ou " infotechnique ». On doit se demander pourquoi cela peut avoir du sens. On peut dire au moins que ces usages de " technologie » concernent des modes d'existence originaux (le vivant, l'information) structurés d'une certaine manière et selon une logique qui leur est propre. Ce sont des domaines de réalité qui possèdent en eux quelque chose comme du logos et qui mettent en œuvre des capacités de reproduction et de transmission. De même, quand on parle de la "sociologie d'un quartier ", d'une " physiologie fragile " ou de la " biologie surprenante des espèces des profondeurs océaniques ", on a certes à faire à des abus de langage qui tendent à réifier le discours (la "-logie ") en le confondant avec l'objet du discours, mais pas seulement. On désigne aussi un certain régime de fonctionnement, un lien signifiant entre le mode d'existence et le mode de connaissance.

Serait ainsi technologique toute pensée qui énonce un problème des rapports (de connexion comme de décalage) entre technè et logos, technicité et discursivité ${ }^{109}$, avec différents gradients de " décollement » ou de couplage de l'objet avec la connaissance. La technologie peut alors se confondre avec son objet et s'en détacher, se décontextualiser et se recontexualiser, traduire, trahir, transduire. On pourrait aussi faire l'hypothèse que les sens de technologie se redéterminent en partie suivant les domaines (biotechnologies, technologies cognitives, nanotechnologies, etc.), si bien qu'au lieu d'une technologie générale et de technologies spéciales (suivant la partition

109. C'est le cas du motif des rapports entre le geste et la parole, central chez André Leroi-Gourhan, ou de celui du rapport des pratiques discursives et non-discursives, ou du dicible et du visible, chez Michel Foucault. 
de Beckmann), il faudrait penser plutôt des technologies génériques ${ }^{110}$ et transductives (qui se retraduisent différent d'un domaine à un autre) ${ }^{111}$.

Que peut la technologie au sens où nous l'entendons contre la conception dominante des GAFAM $^{112}$ aujourd'hui ? Non pas s'y opposer, mais la démultiplier. La diversification des sens de technologie relève d'une stratégie darwinienne de base : plus on multiplie les petites variantes, plus l'une d'elles a une chance de survivre face au gros, qui fera son temps. Il s'agit de réaffirmer que ne sont pas technologiques que les objets «smart» embarquant de l'intelligence artificielle, mais aussi bien l'outil qui invite le geste, la ceinture de sécurité ou la clé berlinoise ${ }^{113}$. Tous portent en eux et transmettent aux utilisateurs des savoirs sociaux et des codes de conduite. Les objets quotidiens et low-tech sont tout aussi constitutifs de nos savoirs, savoir-faire et savoir-vivre que ceux des nouvelles technologies ${ }^{114}$.

Que faire du caractère "idéologique » du sens actuel de technologie ${ }^{115}$ ? D'abord rappeler que le sens ancien de technologie n'est pas moins "idéologique " (mercantiliste, étatiste, progressiste, anti-luddite...) que le sens

110. Voir Terry Shinn, Bernward Joerges, "The transverse science and technology culture: Dynamics and roles of research-technology", Social Science Information, vol. 41, $\mathrm{n}^{\circ} 2,2002$, p. 207-251.

111. Voir Gilbert Simondon, "La mentalité technique " (1961), in Gilbert Simondon, Sur la technique (1953-1983), Paris, PUF, 2014, p. 295-313. Nous pensons, sans avoir la place de le développer ici, qu'il y a chez Simondon de nombreuses ressources pour un pluralisme cohérent de la technologie. Outre l' "allagmatique " (interscience des opérations ou "cybernétique universelle ») évoquée plus haut, la technologie prend chez Simondon une multitude de sens dont l'étude des schèmes de fonctionnement des objets techniques (" technologie générale " ou " mécanologie "), la psychosociologie et l'esthétique de la technicité ou techno-esthétique, mais aussi les schèmes de fonctionnement eux-mêmes, qui sont dits " technologiques " et non simplement " techniques " quand ils articulent plusieurs ordres de grandeur. Chez Simondon, les relations multiscalaires (entre échelles micro/méso/macro, etc.) sont tout autant des opérations réelles que des modes de connaissance. Sur les manières d'articuler ces sens multiples, voir les synthèses provisoires de Vincent Bontems, "Le cycle de la technologie chez Simondon ", Cahiers Simondon, n 6, 2015, p. 7-27 et de Sacha Loeve, «Du récit au design, et retour (des modes de résolution du problème de l'unité de la technologie chez Simondon) ", in Vincent Bontems (dir.), Gilbert Simondon ou linvention du futur. Colloque de Cerisy, Paris, Klincksieck, 2016, p. 113-124.

112. Google Apple Facebook Amazon Microsoft.

113. Bruno Latour, La clef de Berlin et autres leçons d'un amateur de sciences, Paris, La Découverte, 1993.

114. David Edgerton, Shock of the old: Technology and global history since 1900, Londres, Profile Books, 2011; traduction française : Quoi de neuf? Du rôle des techniques dans l'histoire globale, Paris, Seuil, 2013.

115. L. MarX, art. cit, 2010. 
actuel, il l'est différemment. La technologie est une notion normative, chargée de valeurs, qu'il s'agit d'expliciter et de mettre en débat.

Que faire de son flou sémantique voire de son indétermination? Le considérer comme une chance, une flexibilité interprétative propice aux interprétations nouvelles; non pas imposer " la » bonne re-sémantisation contre la technologie des GAFAM, non pas réactiver une technologie unifiée et systématique, mais pluraliser le logos de technè ; non pas se contenter d'un pluralisme descriptif se bornant à constater la multiplicité des acceptions et des usages de la notion de technologie, mais oser un pluralisme normatif consistant à multiplier activement les sens de technologie et à assumer, contre tout relativisme sceptique, les parti pris et les conflits de valeur que cristallise la technologie.

Que faire enfin, de la confusion entre l'épistémologie (modes de connaissance) et l'ontologie (modes d'existences) commise par la signification actuelle de technologie ? Loin d'induire une perte d'intelligibilité, nous pensons que celle-ci est au contraire une chance pour une philosophie du schème qui traverse justement ce partage ${ }^{116}$. L'intérêt de la notion de schème est qu'elle permet de penser la connaissance comme opération, comme mode de relation réalisant un fonctionnement. Un schème technique est à la fois ce par quoi un dispositif fonctionne et la manière de le connaître, analogie d'opération et analogie de connaissance.

\section{Épilogue : un phénix peut en cacher tant d'autres}

La technologie est souvent comparée à un phénix. Elle se présente comme une succession de tentatives, d'instaurations, d'échecs, d'oublis, de réinventions - comme un phénix épistémique renaissant sans cesse de ces cendres sous un nouveau plumage.

Pour autant, la métaphore peut donner lieu à plusieurs usages. Tantôt, elle sert à insister sur la continuité retrouvée par-delà les ruptures et les interruptions, et témoigne de la pertinence d'une science qui ne demande qu'à être reconnue et développée, comme l'affirme par exemple F. Sigaut. Tantôt, elle permet d'insister sur la nouveauté, sur la singulière propension 
de la technologie à ressurgir sans cesse pour qualifier de nouvelles pratiques $^{117}$. La métaphore insiste tantôt sur la bête, tantôt sur son plumage.

On pourrait proposer une troisième manière d'envisager le phénix technologique, à partir du constat que la différence entre ses configurations anciennes et contemporaines réside dans l'apport désormais incontournable de plus de cinquante ans de recherches historiennes sur la technologie. Une différence qu'il ne faut certes pas absolutiser, car on est bien loin de tout connaître des " technologies " passées. Simplement, on ne peut plus les ignorer, de sorte qu'il devient désormais possible de faire l'épistémologie des projets de technologie passés (une épistémologie historique des technologies en quelque sorte) et d'en tirer des leçons (concernant notamment les conditions de possibilités et les limites d'une connaissance technologique) pour les intégrer à de nouvelles pratiques technologiques. Ainsi, les phénix technologiques qui renaîtront ne pourront plus être aussi oublieux des précédents que ces derniers le furent des phénix d'antan. Peut-être la technologie doit-elle demeurer un phénix, au sens où elle demande moins à être unifiée et fixée dans un paradigme qu’à être reconnue dans sa capacité à renaître sous de multiples formes, partout où les techniques font penser.

117. G. Carnino, L. Hilaire-Pérez, art. cit., 2017, p. 31 et suivantes. 


\section{Auteurs}

Sacha Loeve est philosophe des techniques, maître de conférences à l'université Jean Moulin Lyon 3 et membre de l'Institut de recherches philosophiques de Lyon (IRPhiL). Ses recherches sont centrées sur les objets comme nœuds d'articulation entre pratiques, savoirs et récits, et posent notamment la question d'une technologie aujourd'hui, en sciences humaines comme en technosciences. Il a récemment codirigé l'ouvrage French philosophy of technology. Classical readings and Contemporary approaches (Springer, 2018) avec Xavier Guchet et Bernadette Bensaude Vincent, avec laquelle il fait aussi paraître une biographie du carbone (Seuil, 2018).Contact : sacha.loeve@univ-lyon3.fr.

Timothée Deldicque est doctorant en histoire et philosophie des techniques au Centre Alexandre-Koyré (EHESS/CNRS/MNHN), doctorant associé au laboratoire Connaissance, organisation et systèmes techniques (COSTECH) de l'université de technologie de Compiègne (UTC) et membre du Groupement d'intérêt scientifique "Unité des technologies et des sciences de l'homme » (GIS UTSH). Ses recherches portent sur la construction du problème public de léducation technologique dans la seconde moitié du xxe siècle en France. Il a coécrit « Éléments d'anthropologie des sciences humaines et sociales en univers technologique " avec Sacha Loeve et Pierre Steiner dans le cadre du projet FEDER HomTech (" Sciences de l'homme en univers technologique », 2015-2017). Voir http:// www.costech.utc.fr/CahiersCOSTECH/spip.php?rubrique10. Contact : timothee. deldicque@gmail.com. 